\title{
Rora Regulates Neutrophil Migration and Activation in Zebrafish
}

\section{OPEN ACCESS}

Edited by:

Janos G. Filep,

Université de Montréal, Canada

Reviewed by:

Cyrielle Billon,

St. Louis College of Pharmacy,

United States

Ives Charlie-Silva,

University of São Paulo, Brazil

*Correspondence:

Qing Deng

qingdeng@purdue.edu

tORCID:

Christopher J. Staiger orcid.org/0000-0003-2321-1671

${ }^{\ddagger}$ Present addresses: Alan Y. Hsu,

Department of Lab Medicine, The Stem Cell Program, Boston

Children's Hospital, Boston, MA,

United States; Department of Pathology, Harvard Medical School, Dana-Farber/Harvard Cancer Center,

Boston, MA, United States

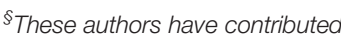
equally to this work and share the first authorship

Specialty section:

This article was submitted to Molecular Innate Immunity, a section of the journal

Frontiers in Immunology

Received: 09 August 2021 Accepted: 04 February 2022 Published: 04 March 2022

Citation:

Hsu AY, Wang T, Syahirah $R$, Liu S, Li K, Zhang W, Wang J,

Cao Z, Tian S, Matosevic S,

Staiger JC, Wan J and Deng Q (2022) Rora Regulates Neutrophil Migration and Activation in Zebrafish.

Front. Immunol. 13:756034. doi: 10.3389/fimmu.2022.756034

\begin{abstract}
Alan Y. Hsu ${ }^{1 \neq \S}$, Tianqi Wang ${ }^{1 \S}$, Ramizah Syahirah ${ }^{1 \S}$, Sheng $\mathrm{Liu}^{2,3}$, Kailing $\mathrm{Li}^{3,4}$, Weiwei Zhang ${ }^{1}$, Jiao Wang ${ }^{5}$, Ziming Cao ${ }^{1}$, Simon Tian ${ }^{1}$, Sandro Matosevic ${ }^{5,6}$, Christopher J. Staiger ${ }^{1,7+}$, Jun Wan ${ }^{2,3,4,8}$ and Qing Deng ${ }^{1,6,9 *}$

${ }^{1}$ Department of Biological Sciences, Purdue University, West Lafayette, IN, United States, ${ }^{2}$ Department of Medical and Molecular Genetics, Indiana University School of Medicine, Indianapolis, IN, United States, ${ }^{3}$ Collaborative Core for Cancer Bioinformatics, Indiana University Simon Cancer Center, Indianapolis, IN, United States, ${ }^{4}$ Department of BioHealth Informatics, Indiana University School of Informatics and Computing, Indiana University - Purdue University Indianapolis, Indianapolis, IN, United States, ${ }^{5}$ Department of Industrial and Physical Pharmacy, Purdue University, West Lafayette, IN, United States, ${ }^{6}$ Purdue University Center for Cancer Research, Purdue University, West Lafayette, IN, United States, 7 Department of Botany and Plant Pathology, Purdue University, West Lafayette, IN, United States, ${ }^{8}$ Center for Computational Biology and Bioinformatics, Indiana University School of Medicine, Indianapolis, IN, United States, 9 Purdue Institute for Inflammation, Immunology, and Infectious Disease, Purdue University, West Lafayette, IN, United States
\end{abstract}

Neutrophil migration and activation are essential for defense against pathogens. However, this process may also lead to collateral tissue injury. We used microRNA overexpression as a platform and discovered protein-coding genes that regulate neutrophil migration. Here we show that miR-99 decreased the chemotaxis of zebrafish neutrophils and human neutrophil-like cells. In zebrafish neutrophils, miR-99 directly targets the transcriptional factor RAR-related orphan receptor alpha (roraa). Inhibiting ROR $\alpha$, but not the closely related ROR $\gamma$, reduced chemotaxis of zebrafish and primary human neutrophils without causing cell death, and increased susceptibility of zebrafish to bacterial infection. Expressing a dominant-negative form of Roro or disrupting the roraa locus specifically in zebrafish neutrophils reduced cell migration. At the transcriptional level, ROR $\alpha$ regulates transmembrane signaling receptor activity and protein phosphorylation pathways. Our results, therefore, reveal previously unknown functions of miR-99 and ROR $\alpha$ in regulating neutrophil migration and anti-microbial defense.

Keywords: miR-99, RAR-related orphan receptor, chemotaxis, innate immunity, zebrafish

\section{INTRODUCTION}

Inflammation is essential for the restoration of tissue homeostasis after injury and infection. Neutrophils, the primary effector cells of acute inflammation, are the first to infiltrate inflammation sites. They combat disease by phagocytosing foreign particles and secreting cytokines, antimicrobial molecules, and chromatin while contributing to collateral tissue injury (1). Under inflammatory conditions, neutrophils are a heterogeneous population with pro-and antiinflammatory phenotypes that cooperate to resolve inflammation $(2,3)$. In line with their multifaceted roles, neutrophils contribute to dangerous, chronic inflammatory conditions in humans, including those associated with tumors, chronic obstructive pulmonary disease, arthritis, and adipose inflammation. On the other hand, neutrophil activity is beneficial to wound healing, infarction repair, and inflammatory bowel diseases (4). Enhancing, inhibiting, or 
restoring neutrophil functions are attractive therapeutic strategies (5). A better understanding of the neutrophilintrinsic mechanisms that regulate neutrophil migration will have broad translational importance in preventing and treating a wide range of inflammation-related diseases. There are challenges associated with studying terminally differentiated neutrophils; namely, only a limited set of genetic tools are available to examine the plasticity of the cells in vivo. To address this challenge, we use zebrafish, a genetically tractable vertebrate model with a well-conserved innate immune system (6). Adaptive immunity is morphologically and functionally mature only after 4-6 weeks postfertilization. Zebrafish embryos acquire microbiota when hatching at 2-3 days postfertilization. Therefore zebrafish embryonic provides a unique tool to investigate innate immunity without the impact from the adaptive immune system (7).

MicroRNAs are small RNA molecules of 22-24 nt that regulate homeostasis in health and disease (8). MicroRNAs primarily suppress target gene expression by binding microRNA seed sequences (nucleotides 2-8) to partially complementary sequences in their transcripts' 3' untranslated regions. MicroRNA "mimics" and "inhibitors" are emerging as next-generation therapeutics because of their ability to modulate a network of genes $(9,10)$. In addition, microRNAs are being used as tools to discover novel regulators of biological processes. Through a phenotypic screen in zebrafish, we identified nine microRNAs that, when overexpressed, suppressed neutrophil migration (11). Among the positive hits, dre-miR-99-1 significantly reduced neutrophil chemotaxis. The current study characterized miR-99 substrates and discovered an unexpected role of the RAR-related orphan receptor $(\mathrm{ROR} \alpha)$ in regulating neutrophil migration.

$\mathrm{ROR} \alpha$ regulates embryonic development, metabolism, circadian rhythm, and inflammation (12-14). In the immune system, recent studies suggested prominent role of $\operatorname{ROR} \alpha$ in regulating T cell and ILC2 development $(15,16)$ and immune cell functions, including $\mathrm{T}$ regulatory cells in allergic skin inflammation (17), Th17 driven inflammatory disorders (18, 19), survival of CD4+ T cells in colitis (20) and lung infection (21), ant-tumor immunity of liver-resident natural killer cells/ ILC1s (22), macrophage activation in LPS-induced septic shock (23) and diet-induced obesity (24), and ILC3 driven fibrosis (25). However, the role of ROR $\alpha$ in regulating neutrophil function was not previously known. Our results that $\operatorname{ROR} \alpha$ is required for neutrophil migration and anti-microbial defense complement the previous works and provide a complete understanding and foundation for $\operatorname{ROR} \alpha$ targeting drugs in treating various inflammatory conditions.

\section{MATERIALS AND METHODS}

\section{Generation of Transgenic Zebrafish Lines}

The zebrafish experiment was conducted in accordance with internationally accepted standards. The Animal Care and Use Protocol was approved by The Purdue Animal Care and Use
Committee (PACUC), adhering to the Guidelines for the use of Zebrafish in the NIH Intramural Research Program (protocol number: 1401001018). Zebrafish roraa gene was cloned from zebrafish mRNA using SuperScript III RT-kit (Invitrogen $\# 18080044$ ) and amplified with the following primers. zroraa $\mathrm{wt}+: 5^{\prime}$ - aaaacccggtcctatgcatatgatgtattttgtgatttcagctatgaaagct- $3^{\prime}$; zroraa wt-: $5^{\prime}$-ctgattatgatctagactacccgtcaacgggcat-3; zroraa LBD deletion $\mathrm{DN}+$ : $5^{\prime}$ - aaaacccggtcctatgcatatgatgtatttgtgatttcag ctatgaaagctcaaatcg- $3^{\prime}$; zroraa LBD deletion DN -: 5' - ctga ttatgatctagagtccaggccggattgatcagg- 3 and inserted into a Tol2lyzC-mcherry-2A backbone by using infusion cloning kit (Takara \#638920). The construction method for neutrophilspecific Cas9 expression and the guide RNA expression fish lines has been described in our previous study (26). The protospacer sequences for targeting dre-roraa in this research are $5^{\prime}$-taattcctgcaagatctg-3'; $5^{\prime}$-gcacgttatcacgccataa- $3^{\prime}$, both guide RNAs target on dre roraa exon2. Microinjections of fish embryos were performed by injecting $1 \mathrm{nl}$ of a mixture containing $25 \mathrm{ng} / \mu \mathrm{l}$ plasmids and $35 \mathrm{ng} / \mu \mathrm{l}$ tol 2 transposase mRNA in an isotonic solution into the cytoplasm of embryos at the one-cell stage. The stable lines were generated as previously described (27). At least two founders (F0) for each line were obtained. Experiments were performed using F2 larvae produced by F1 fish derived from multiple founders to minimize the artifacts associated with random insertion sites.

\section{RNA Seq}

Kidney marrow was dissected from 3 adults from $\operatorname{Tg}(l y z C$ : $m i R$ 99-1-dendra2) $)^{\text {pu25 }}, \operatorname{Tg}(\text { lyzC: vector-dendra2 })^{\text {pu7 }}, \operatorname{Tg}(\operatorname{lyz} C$ : $m$ cherry-2a $)^{p u} 32$ and $\operatorname{Tg}(m$ cherry-2a-ROR $\alpha \mathrm{DN}){ }^{p u 33}$ and neutrophils were sorted using FACS. Total RNA was extracted using RNeasy Plus Mini Kit (Qiagen \#74104). RNAseq was performed at The Center for Medical Genomics at Indiana University School of Medicine. Samples were polyA enriched and sequenced with Illumina HiSeq 4000 ultra-low with reads ranging from $37 \mathrm{M}$ to $44 \mathrm{M}$. The RNA-seq aligner from the STAR (v2.5) (28) was employed to map RNA-seq reads to the reference genome, zebrafish (GRCz11), with previously the following parameter: “-outSAMmapqUnique 60". Uniquely mapped sequencing reads were assigned to genes using featureCounts (from subread v1.5.1) (29) with the following parameters: “-p -Q 10 ". The genes were filtered for further analysis if their count per million (CPM) of reads was less than 0.5 in more than three samples. The method of a trimmed mean of $\mathrm{M}$ values (TMM) was adopted for gene expression normalization across all samples, followed by differential expression analysis between different conditions using edgeR (v3.20.8). Differentially expressed gene was determined for the comparison if its false discovery rate (FDR) adjusted p-value was less than 0.05 and the amplitude of fold change (FC) was larger than linear 2 -fold. The functional analysis was performed on DEGs of our interest with a cutoff of FDR $<0.05$ to identify significantly over-represented Gene Ontology (GO) and/or KEGG pathways by using the DAVID (30). To compare the down-regulated DEG set in roro DN neutrophil to the published THP-1/HUVEC ROR $\alpha$ CHIPseq data, the genes from CHIP-seq result were converted to their orthologues in zebrafish. The gene duplicates after the 
conversion from human cells were removed. The zebrafish genes not having the orthologues to human were also removed before comparison.

\section{Quantitative RT-PCR}

Total RNA was purified using MiRVANA miRNA purification kit (ThermoFisher) for miRNA or RNeasy Plus Mini Kit (Qiagen \#74104) for mRNA. MicroRNAs were reverse transcribed with Universal cDNA Synthesis Kit II (Exiqon). MicroRNA RT-qPCR was performed with ExiLENT SYBR ${ }^{\circledR}$ Green master mix (Exiqon), and mRNA with one-step RT-qPCR was performed with SuperScript ${ }^{\circledR}$ III Platinum ${ }^{\circledR}$ SYBR $^{\circledR}$ Green kit (Invitrogen) using LightCycler ${ }^{\circledR} 96$ Real-Time PCR System (Roche Life Science). The specificity of the primers was verified with a single peak in the melt curve. The relative fold change with correction of the primer efficiencies was calculated following instructions provided by Real-time PCR Miner (http://ewindup. info/miner/data_submit.htm) and normalized to U6. miRNA Primers used in this study were purchased from Exiqon. The relative fold change with correction of the primer efficiencies was calculated following instructions provided by Real-time PCR Miner and normalized to rpl32. Primers: dre-grn1+: $5^{\prime}-$ aacccagccagcaagatg-3', dre-grn1-: 5'-ccaccgggatagacagatca-3', dre-fabp7a+: $5^{\prime}$-cagcagacgatagacatgtgaagt- $3^{\prime}$, dre-fabp7a-: $5^{\prime}$ tcccacctctgaacttggac- $3^{\prime}$, dre-illb+: $5^{\prime}$-atgctcatggcgaacgtc- $3^{\prime}$, dreil1b-: $5^{\prime}$-tggttttattgtaagacggcact- $3^{\prime}$, dre-tgfbr $1+: 5^{\prime}$-tgcaacaa gaacccaaaagtt- $3^{\prime}$, dre-tgfbr1-: $5^{\prime}$-gggactcatagactggggttc- $3^{\prime}$, dreroraa + : $5^{\prime}$-gaatgatcagatagtgcttctcaaag- $3^{\prime}$, dre-roraa-: $5^{\prime}$-cacggca cattctgacga- $3^{\prime}$, dre-rorc+: $5^{\prime}$-tcttttcctatccaacctctctaca- $3^{\prime}$, drerorc-: $5^{\prime}$-gagtggtctctttatgtgagcgta- $3^{\prime}$, dre-rpl32+: $5^{\prime}$ - tcagtctgacc gctatgtcaa-3'; dre-rpl32-: 5' - tgcgcactctgttgtcaatac-3'; dre-ef1a $+: 5^{\prime}$ - tgccttcgtcccaatttcag-3'; dre-ef1a-:5' - taccctccttgcgctcaatc3'; dre-roraa-overexpression+:5' - tcaggcatccattatggcg-3'; dreroraa-overexpression-:5' - cggtcaatcaggcagttctt-3'; dre-arhgap $17 b+: 5^{\prime}$ - ttggcggatgaagaggattc-3'; dre-arhgap 17b-:5'- ccaat cgcacattttccagg-3'; dre-ptk2ba+:5' - attacatgcagcacaacgcc-3'; dreptk2ba-:5'-ccagacccacatctttctcca-3'; dre-mhclzba+:5' - gaagattc ccaaacagcactgg-3'; dre-mhc1zba-:5' -cgttccatcagaatgttgacgttc- $3^{\prime}$; dre-tcerg11+:5'-aagcagagaacagcggataag-3'; dre-tcerg11-:5' ttagcctctgggtttggattg- 3 '.

\section{Dual-Luciferase Reporter Assay}

zebrafish roraa 3'UTR was amplified from zebrafish genomic DNA with SuperScript III RT-kit (Invitrogen \#18080044) and cloned into psiCHECK2 (Promega) at XhoI and Not I cloning sites with the following primers: zroraa $+: 5^{\prime}$ - taggcgatcgctcga caaggcagctcactaggaacagaactg-3'; zroraa-: 5' - ttgcggccagcggc cggaggacagggaaacgtagctgtac-3'. Mutated 3'UTR constructs were generated using Infusion $\mathrm{HD}$ cloning kit (Clontech) with the following primers: zroraa mut+: 5'- acaaaatgeccattctacaggg taaccactgctgtgg-3'; zroraa mut-: 5'- gaatgggcattttgtcgagcgacg gacgtc-3'. DNA encoding $m i R-99$ or vector was amplified from the construct used for expression in zebrafish and inserted into pcDNA3.1 at the HindIII/XbaI cloning sites using the following primers: pcDNA+: 5;- gtttaaacttaagcttgccaccatggatgaggaaatcgc3'; pcDNA-: 5'-aaacgggecctctagagaccggtaccccgggctgc-3'.

\section{Live imaging}

Larvae at three dpf were settled on a glass-bottom dish, and imaging was performed at $28{ }^{\circ} \mathrm{C}$. Time-lapse spinning disk confocal microscopy (SDCM) was performed with a Yokogawa scanning unit (CSU-X1-A1) mounted on an Olympus IX-83 microscope, equipped with a 20X 0.5 -numerical aperture (NA) UPlanSApo oil objective (Olympus) and an Andor iXon Ultra 897BV EMCCD camera (Andor Technology). GFP and mCherry were excited with $488 \mathrm{~nm}$ and $561 \mathrm{~nm}$ and fluorescence emission collected through 525/30-nm and 607/36-nm filters, respectively, to determine the localization of stable actin in migrating neutrophils in vivo. Images were captured using MetaMorph version 7.8.8.0 software at $10 \mathrm{~s}$ intervals for a total of $5 \mathrm{~min}$. Time-lapse fluorescence images in the head mesenchyme were acquired with a laser-scanning confocal microscope (LSM710, Zeiss) with a Plan-Apochromat $20 \times / 0.8$ M27 objective. The fluorescent stacks were flattened using the maximum intensity projection and overlaid with a single slice of the bright-field image. The velocity of neutrophils was quantified using ImageJ with the MTrackJ plugin.

\section{Inflammation Assays in Zebrafish}

Zebrafish wounding and infection were performed as described (31). Briefly, three dpf larvae were amputated posterior to the notochord or inoculated with $P$. aeruginosa (PAK) into the left otic vesicle or the vasculature at $1000 \mathrm{CFU} / \mathrm{embryo}$. The larvae were fixed in $4 \%$ paraformaldehyde at one h post-wounding or -infection. Neutrophils were stained with Sudan Black, and the number at the indicated regions was quantified.

\section{Generation of Stable HL-60 Cell Lines}

HL-60 cells were obtained from ATCC (CCL-240), and cultured using RPMI-1640 with HEPES supplemented with 10\% FBS and sodium bicarbonate. The lentiviral backbone pLIX_403 was a gift from David Root (Addgene plasmid \# 41395). The DNA sequence flanking mir-99 was cloned from HL-60 cell genomic DNA and cloned into a backbone containing dendra2. The miRNA and dendra2 reporter were then cloned into pLIX_403 vector using the NheI/AgeI sites with primer set: pLIX-mir+: 5' tggagaattggctagcgccaccatggatgaggaaatcgc-3', pLIX-mir-: 5' catacggataaccggttaccacacctggctgggc-3'. Stable HL-60 cell lines were generated as described $(32,33)$. Briefly, HEK-293 cells were transfected with pLIX_403 or pLKO.1, together with VSVG and CMV8.2 using lipofectamine 3000 (ThermoFisher). The viral supernatant was harvested on day three and concentrated with Lenti-X concentrator (Clontech 631232). HL-60 cells were infected with concentrated lentivirus in complete RPMI medium supplemented with four $\mu \mathrm{g} / \mathrm{ml}$ polybrene (Sigma TR-1003-G) at $2500 \mathrm{~g}$ for $1.5 \mathrm{~h}$ at $32^{\circ} \mathrm{C}$ and then selected with one $\mu \mathrm{g} / \mathrm{ml}$ puromycin (Gibco A1113803) to generate stable lines.

\section{Flow Cytometry}

Cells were stained with Alexa Fluor 647 conjugated CD11b (neutrophil differentiation marker, Biolegend, 301319, clone IV-M047, 2 ul to $1 \times 10^{6}$ cells) or the isotype control antibody (Biolegend, 400130, clone MOPC-21, 2 ul to $1 \times 10^{6}$ cells), RUO 
AnnexinV (apoptosis marker, BD, 563973, 5ul to $1 \times 10^{6}$ cells) and RUO propidium iodide (dead cell marker, BD, 556463, 5ul to $1 \times 10^{6}$ cells). Cells were stained in staining buffer (1\% BSA, $0.1 \% \mathrm{NaN} 3$ ) and incubated on ice for 1 hour, washed three times with staining buffer, and resuspended in suitable volumes. Cell cycle profiling was obtained using Vybrant ${ }^{\mathrm{TM}}$ DyeCycle ${ }^{\mathrm{TM}}$ Ruby Stain (Thermo \#V10309). Fluorescence intensity was collected using BD LSR Fortessa. Results were analyzed with Beckman Kaluza 2.1 software.

\section{Transwell Migration Assay}

Transwell assays were performed as described (32). Briefly, $2 \times 10^{6}$ cells/ml differentiated HL-60 cells were resuspended in HBSS with $0.5 \% \mathrm{FBS}$ and $20 \mathrm{mM}$ HEPES. $100 \mu \mathrm{l}$ were placed into a 6.5 $\mathrm{mm}$ diameter $3 \mu \mathrm{m}$ pore size transwell insert (Corning\#3415) and allowed to migrate for two $\mathrm{h}$ at $37^{\circ} \mathrm{C}$ towards $100 \mathrm{nM}$ fMLP in $500 \mu \mathrm{l}$ of HBSS in a 24-well plate. Loading controls were done by directly adding $100 \mu \mathrm{l}$ of cells to $400 \mu \mathrm{l}$ of HBSS. Cells that migrated to the lower chamber were released with 0.5 M EDTA and counted using a BD LSRFortessa flow cytometer with an acquisition time of $30 \mathrm{~s}$. The counts were normalized with the total numbers of cells added to each well the data was then gated for live cells and analyzed with Beckman Kaluza 2.1 software.

\section{NETosis Assay}

Differentiated HL-60 cells (dHL60) were resuspended in HBSS in $20 \mathrm{mM}$ HEPES with $0.5 \%$ FBS (mHBSS) and allowed to attach to fibrinogen-coated slides for $30 \mathrm{~min}$ at $37^{\circ} \mathrm{C}$. Neutrophil extracellular traps (NETs) were induced with $50 \mathrm{nM}$ PMA (sigma \#P1585) in HBSS for $4 \mathrm{~h}$ at $37^{\circ} \mathrm{C}$. NETs were enumerated using cell-permeable Hoechst 33258 at $1 \mu \mathrm{g} / \mathrm{ml}$ (Thermo \#62249) and cell impermeable Sytox Green at $1 \mu \mathrm{M}$ (Thermo \#S7020). Images were acquired using Lionheart FX Automated Microscope (Biotek) 10x phase objective, Plan Fluorite WD 10 NA 0.3 (1320516). The percentage of cells forming NETs was calculated by dividing the number of Sytox Green-positive cells by that of the Hoechst-positive cells.

\section{ROS Assay}

Amplex ${ }^{\mathrm{TM}}$ Red Hydrogen Peroxide/Peroxidase Assay Kit (Thermo \#A22188) was used to detect extracellular ROS. dHL60 cells $(20,000$ cells per well) were incubated with $0.1 \mu \mathrm{g} /$ mL of PMA (sigma \#P1585) in 96 well black/clear bottom plate (Thermo \#165305). ROS reading was taken 30 - 45 minutes after stimulation using Synergy Neo2 (BioTek) at Ex/Em of 571/585 $\mathrm{nm}$. The amount of hydrogen peroxide release was calculated by comparing to standards prepared.

\section{Phagocytosis Assay}

BioParticles from pHrodo ${ }^{\mathrm{TM}}$ Green BioParticles ${ }^{\circledR}$ Conjugates (Thermo \# P35366) were reconstituted and sonicated for 5 minutes at $10 \%$ amplitude with 10 seconds intervals. dHL60 cells $\left(5 \times 10^{5}\right)$ were resuspended in $100 \mathrm{uL}$ of BioParticle suspension and incubated at $37^{\circ} \mathrm{C}$ for 1 hour. The reaction is stopped by placing on ice before running flow cytometry to quantify the mean FITC intensity in the cell population. Cells with beads placed on ice for 1 hour were used as the negative control.

\section{Inhibitor Treatment for Zebrafish Larvae}

ROR inhibitors SR3335 (Cayman \# 12072), SR2211 (Cayman \# 11972), SR1001 (Cayman \# 10922), and VPR-66 (Novus \# NBP2-29335) were dissolved in DMSO to make a $100 \mathrm{mM}$ stock, then further diluted in E3 to working concentrations (10-100 $\mu \mathrm{M}$ for recruitment and motility assays, and indicated concentration for survival assays). For neutrophil recruitment and random motility assays, larval were pretreated with the inhibitor for one $\mathrm{h}$ before experimental procedures. For survival assays, larval were pretreated and kept in the inhibitors from 1-hour post-infection.

\section{Primary Neutrophil Isolation and Chemotaxis Assay}

Primary human neutrophils were obtained from the peripheral blood of healthy adult donors, collected under approval by Purdue University's Institutional Review Board (IRB). Primary human neutrophils were isolated with Milteny MACSxpress Neutrophil Isolation Kit (Macs \# 130-104-434), and RBC lysed

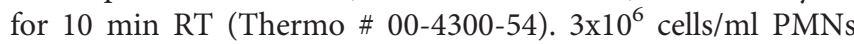
were incubated in HBSS with $0.1 \%$ FBS and 20mM HEPES with or without respected inhibitors for 1 hour. Cells were then mixed 3:1 with matrigel (corning \# 356234) and loaded into collagencoated IBIDI chemotaxis $\mu$-slides (ibidi \# 80326) and incubated at $37^{\circ} \mathrm{C}$ for 20 minutes to allow 3D matrix formation. $15 \mu \mathrm{l}$ of $1000 \mathrm{nM}$ fMLP was loaded into the right reservoir yielding a final fMLP concentration of $187 \mathrm{nM}$. Chemotaxis was recorded every 1 min for two h, with a Lionheart FX Automated Microscope (Biotek) 10x phase objective, Plan Fluorite WD 10 NA 0.3 (1320516). Chemotaxis tracking was done with the MTrackJ image J plugin and plotted in Prism 6.0 (GraphPad). Cell viability of primary human neutrophils treated with ROR inhibitor SR3335 (Cayman \# 12072) was measured by staining with propidium iodide and analyzed using flow cytometry.

\section{Immunoblot Assay of Primary Human Neutrophils}

$1.5 \times 10^{6}$ cells $/ \mathrm{ml}$ of primary human neutrophils were resuspended in DPBS. They were lysed with ice-cold lysis solution in a 1:1 ratio (20\% TCA, $40 \mathrm{mM} \mathrm{NaF}$, and $20 \mathrm{mM} \beta$-glycerophosphate). Cells were incubated on ice for 1-2 hours and then pelleted down. The protein pellet was washed twice with $0.75 \mathrm{~mL} 0.5 \%$ TCA and resuspended in $30 \mu \mathrm{l}$ of $2 \mathrm{x}$ Laemmli sample buffer (Sigma \#S3401). $15 \mu \mathrm{l}$ of the suspension is loaded per well on a $10 \%$ SDS-PAGE gel. Samples were transferred to polyvinylidene difluoride (PVDF) membrane and blocked with $2.5 \%$ non-fat milk in PBST. Primary antibodies used were ROR $\alpha$ Polyclonal Antibody (Thermo \#PA1812 ) and vinculin (Thermo \#MA5-11690) diluted into 1\% BSA at 1:1000 and 1:5000 ratios, respectively.

\section{Mutational Efficiency Quantification}

To determine the mutation efficiency in $\operatorname{Tg}(\operatorname{LyzC}:$ Cas9, Cry : RFP, $U 6 a / c$ : rora guides, $L y z C: G F P)^{p u 34}$, the roraa loci around the 
sgRNA binding site was amplified by PCR using the following primers: dre roraa,$+ 5^{\prime}$ - cgactggtgacagtgaaaaatc- $3^{\prime}$; dre roraa-, 5' tttagcttcttaccttgcagcc- $3^{\prime}$. PCR products were deep sequenced using WideSeq at the Purdue Genomics Core Facility. Mutation efficiencies were calculated using the CRISPResso2 package (www.github.com/pinellolab/CRISPResso2).

\section{Survival Assay}

Larvae at three dpf were injected with one $\mathrm{nl}$ of $25 \mathrm{ng} / \mathrm{nl}$ LPS or 1000 CFU P. aeruginosa (PAK) into the tail vein and incubated individually in 96-well plates. Survival was tracked for four days or when one group reached $100 \%$ mortality. Representative results of at least three independent experiments ( $n \geq 20$ larvae in each experiment) were shown.

\section{Statistical Analysis}

Statistical analysis was carried out using PRISM 6 (GraphPad). Mann-Whitney test (comparing two groups), Kruskal-Wallis test (when compared to a single group), and Gehan-BreslowWilcoxon test (for survival curve comparison) were used in this study as indicated in the figure legends. Individual $\mathrm{p}$ values are indicated in the figure, with no data points excluded from statistical analysis. One representative experiment of at least three independent repeats is shown. For qPCR, each gene was normalized to a reference gene and compared with the HolmSidak test (individual comparison with paired value), where each p-value and DF were adjusted for respective multiple comparisons.

\section{RESULTS}

\section{miR-99 Overexpression Inhibits Neutrophil Motility and Chemotaxis}

We use the lyzc promoter to drive gene expression, specifically in zebrafish neutrophils $(34,35)$. The microRNA gene was inserted into the intron of a fluorescent reporter gene (Figure 1A) to allow visualization of neutrophils expressing the transgene. To further characterize the zebrafish line $\operatorname{Tg}(\operatorname{lyz} \mathrm{C}$ : $\mathrm{miR}-99-1 /$ dendra2) $)^{\text {pu25 }}$ with this referred to as miR-99, we first isolated neutrophils and performed miRNA RT-qPCR to confirm miR99 overexpression. Compared with the control line that only expresses the vector, the miR-99 level is significantly higher in the miR-99 line (Figure 1B). The two abundant neutrophil miRNAs, miR-223 and let-7e, were used as controls to demonstrate that this overexpression did not disrupt physiological microRNA levels. miR-99 overexpression did not affect the total neutrophil numbers in embryos (Figures 1C, D) but reduced neutrophil recruitment to either the tail injury or ear infection sites (Figures 1E-H). In addition, the spontaneous neutrophil motility in the head mesenchyme was significantly slower upon miR-99 overexpression (Figures 1I, J and Movie 1). We then sought to determine whether miR-99 inhibits human neutrophil migration. Here we used HL-60 cells, a myeloid leukemia cell line that can be differentiated into neutrophil-like cells as our model $(33,36)$. We have developed a platform to express microRNAs in HL-60 cells after cell differentiation (Figure 2A) (33). As expected, MIR-99 overexpression did not affect cell differentiation, cell death, or the expression of two other miRNAs, MIR-223 and LET-7 (Figures 2B-F). The differentiated (dHL-60) cells were then subjected to a transwell assay using N-Formylmethionyl-leucyl-phenylalanine (fMLP) as the chemoattractant. Overexpressing MIR-99, but not the vector control, significantly reduced chemotaxis (Figure 2G), indicating that overexpression of MIR-99 inhibits neutrophil migration in both zebrafish and human cell models. In addition, MIR-99 suppressed other functions in HL-60 cells, including phagocytosis, production of reactive oxygen species, and neutrophil extracellular traps (Figures $2 \mathbf{H}-\mathbf{J}$ ).

\section{miR-99 Suppresses the Expression of RAR-Related Orphan Receptor Alpha ROR $\alpha$}

To understand the underlying molecular mechanism of how miR-99 regulates neutrophil migration, we sequenced the transcriptome of neutrophils expressing miR-99. Since microRNAs generally suppress the expression of target transcripts (37), we focused on the down-regulated differentially expressed genes (DEG), which are associated with pathways in the cell cycle, microtubule-based movement, and DNA damage stimulus (Figures 3A, B and SI Appendix, Dataset S1, S2). Only two genes among the down-regulated DEGs, roraa, and dec1, contain predicted miR-99 binding sites in the 3'UTR (Figure 3C). Roraa is one of the retinoic acid receptor-related orphan receptor (ROR) gene family members in zebrafish. Identical to humans, there are three ROR genes in zebrafish: rora, rorb, and rorc. The protein product of rora, $\mathrm{ROR} \alpha$, functions in metabolic regulation, energy homeostasis, thymopoiesis, and integration of the circadian clock $(38,39)$. The functions of $\operatorname{ROR} \beta$ are mainly unknown. It perhaps acts as a novel regulator in osteogenesis $(26,40)$. ROR $\gamma$ t, the protein product of rorc, guides Th17 cell differentiation and promotes IL-17 production (19). Compared to $\operatorname{dec} 1$, the roraa gene orthologues are more conserved between zebrafish and humans (zebrafish roraa sequence is $89.96 \%$ identical to its human ortholog rora while dec1 in zebrafish is $59.55 \%$ identical to its human orthologue). In addition, $\mathrm{ROR} \alpha$ polymorphisms correlate with different diseases, such as multiple sclerosis, breast cancer, asthma in human patients (41-43). However, whether ROR $\alpha$ plays a regulatory role in neutrophil migration is unknown. Therefore, we chose roraa to follow up. To validate the RNAseq results, we selected four other genes, grn 1, fabp7, tgfb1, illb, and confirmed their reduced transcript levels in the miR-99-overexpressing neutrophils (Figure 3D). To be noted, the expression of roraa, but not its homolog, rorc, was suppressed by miR-99. We then performed dual-luciferase reporter assays to determine whether miR-99 can directly bind to roraa 3'UTR and suppress reporter expression. miR-99 significantly reduced the relative luciferase activity, dependent on the roraa 3'UTRs (Figures 3E, F), indicating that miR-99 can directly target roraa in zebrafish. 


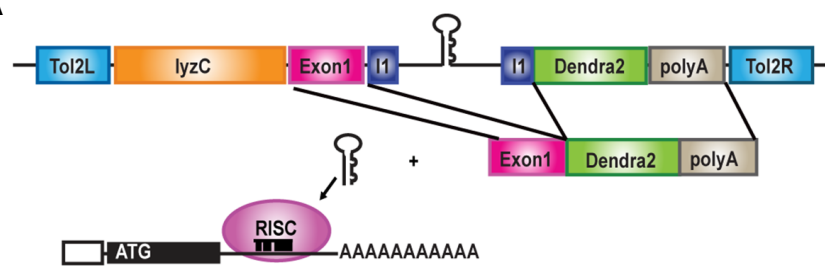

c

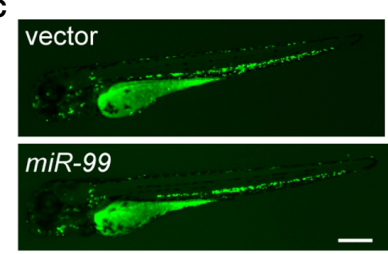

D

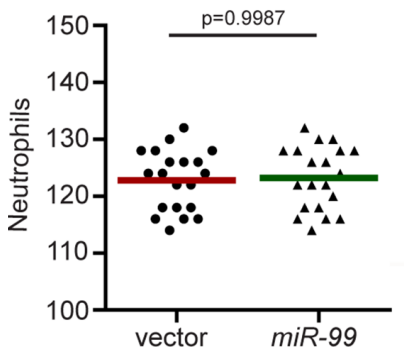

E

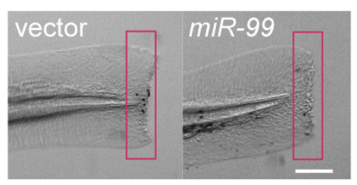

$\mathbf{F}$

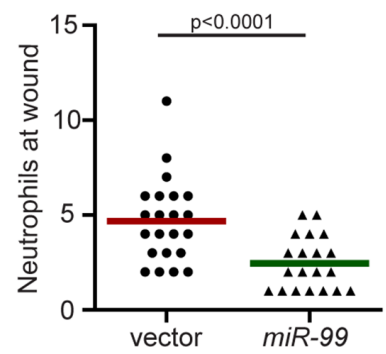

G
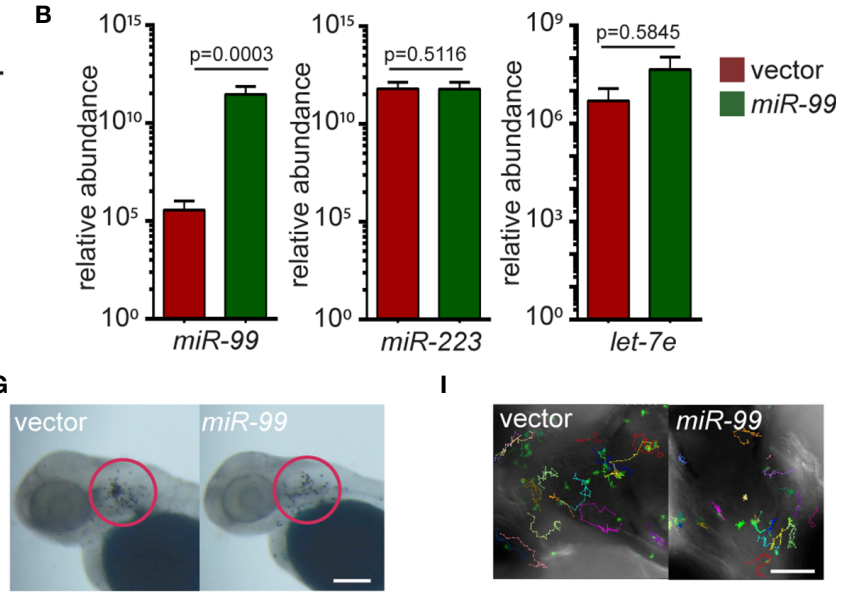

I

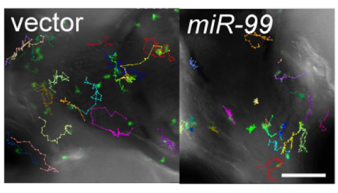

H

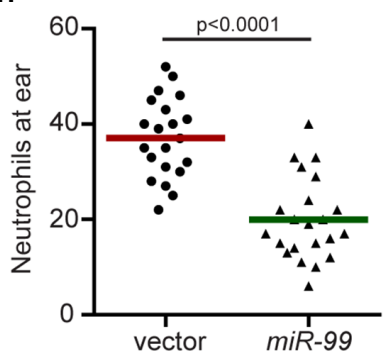

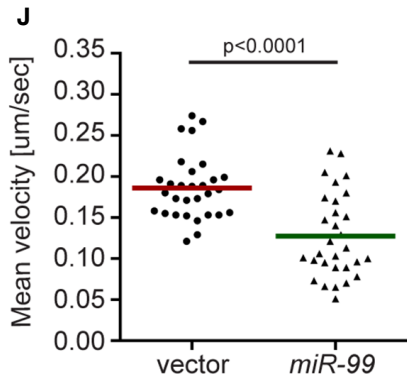

FIGURE 1 | Neutrophil-specific miR-99 overexpression hinders neutrophil recruitment and motility. (A) Schematic of the Tol2-lyzC:miR-99/Dendra2 plasmid, injected into wild-type AB zebrafish embryos to generate the transgenic line Tg(lyzC:miR-99/Dendra2) (miR-99) and Tg(lyzC:Dendra2). (B) The relative expression level of miR-99, miR-223, and let-7e (normalized to U6 expression) in vector and miR-99 lines determined by RT-qPCR, mean \pm s.d. ( $N=3$ biological replicates with ten larvae at each time point in each group). (C, D) Representative images and quantification of total neutrophils in vector and miR-99 lines. Scale bar: 500 um. (E, F) Representative images and quantification of neutrophil recruitment to tail transection site in vector or miR-99 larvae at 1-hour post-injury. Neutrophils in the boxed region were quantified. Scale bar: $100 \mu \mathrm{m}$. (G, H) Representative images and quantification of neutrophil recruitment to localized ear infection in vector or miR-99 larvae at 1-hour post-infection. The infected ear is indicated with the circle. Scale bar: $100 \mu \mathrm{m}(\mathbf{I}, \mathbf{J})$ Tracks and quantification of neutrophil motility in vector or miR-99 larvae. Scale bar: $50 \mu \mathrm{m}$. All experiments were performed with F2 larvae at three dpf. One representative result of three independent experiments was shown ( $\mathrm{n}=20$ ). $P$-value was calculated with unpaired Student's t-test.

\section{Pharmacological Inhibition of Rora Decreases Neutrophil Chemotaxis in Both Zebrafish and Human}

ROR $\alpha$ binds DNA as a monomer and constitutively activates transcription $(44,45)$. Although the endogenous ligands of this receptor are not entirely clear, synthetic ligands can be used to suppress or enhance ROR $\alpha$ activities (46). To test whether Ror $\alpha$ regulates neutrophil migration in homeostasis and inflammation conditions, we used SR3335, an inverse agonist of ROR $\alpha$, SR2211, a ROR $\gamma t$ inverse agonist, and VPR66, an inverse agonist of both ROR $\alpha$ and ROR $\gamma$ t. None of the inhibitors affected zebrafish survival or neutrophil numbers (Figure S1A). Neutrophil motility was explicitly reduced by the ROR $\alpha$, but not the ROR $\gamma$ inhibition (Figures 4A, B and Movie 2). Consistently, neutrophil recruitment to the infected ear (Figures 4C, D) or tailfin amputation sites (Figures 4E, F) was significantly and specifically inhibited by the ROR $\alpha$ inhibitor. Human neutrophils possess a limited transcriptional activity and do not require transcription for chemotaxis $(47,48)$. To ensure that our findings are not restricted to zebrafish, we isolated primary human neutrophils and treated them with the ROR inverse agonists. Similar to our observation made in zebrafish, the inverse agonist of $\operatorname{ROR} \alpha$, but not $\operatorname{ROR} \gamma$, reduced chemotaxis of primary human neutrophils $1 \mathrm{hr}$ after treatment, without causing apoptosis (Figures 4G, $\mathbf{H}$ and Figures S1B-G and Movie 3). ROR $\alpha$ protein is detected in primary human neutrophils (Figure S1H). In addition, the ROR inverse agonists SR3335 suppressed neutrophil chemotaxis in fish in a dose-dependent manner (Figures 4I, J). In summary, we report a previously unrecognized role for $\mathrm{ROR} \alpha$ in regulating neutrophil chemotaxis.

\section{DNA Binding Activity of Rora Is Required for Neutrophil Motility}

Due to the potential off-target effects of the inhibitors, we next sought genetic evidence for $\operatorname{ROR} \alpha$ function in neutrophil migration. ROR $\alpha$ protein consists of one DNA-binding domain and one ligand-binding domain, working as a monomer and activates transcription when binding to the DNA response element. A truncated version of $\mathrm{ROR} \alpha$ lacking 
A

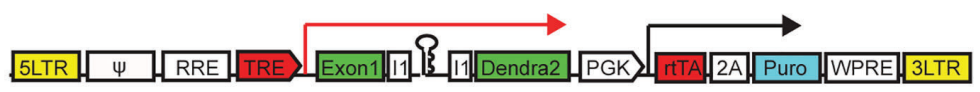

C

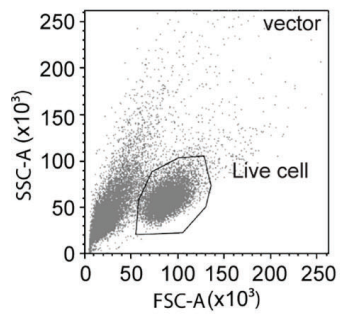

D

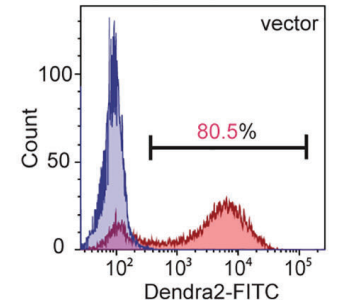

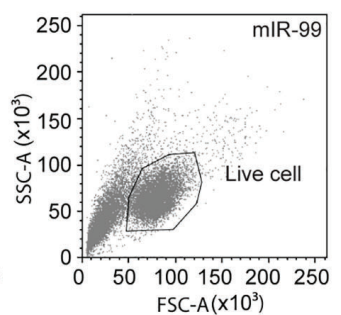

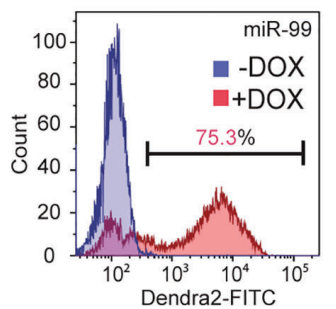

E

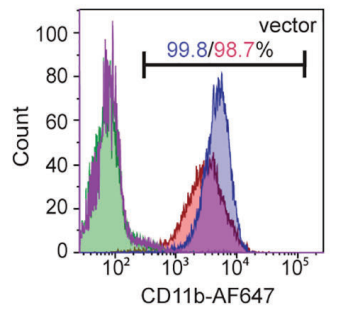

F

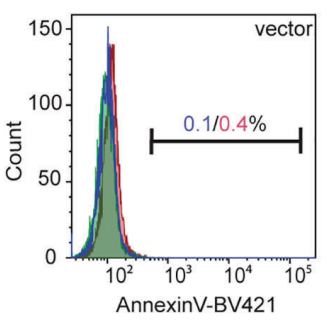

I

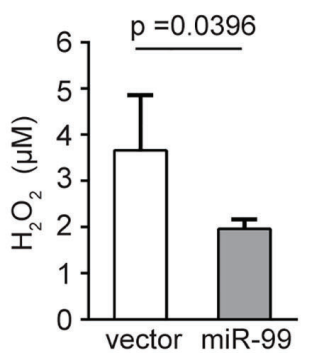

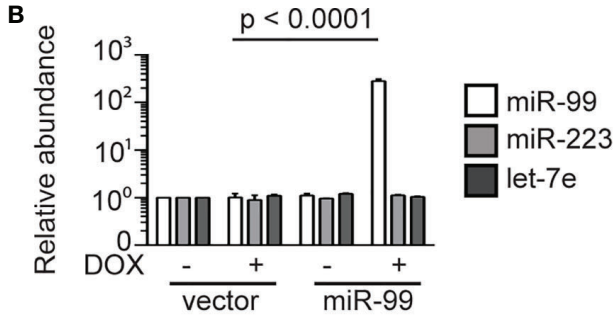
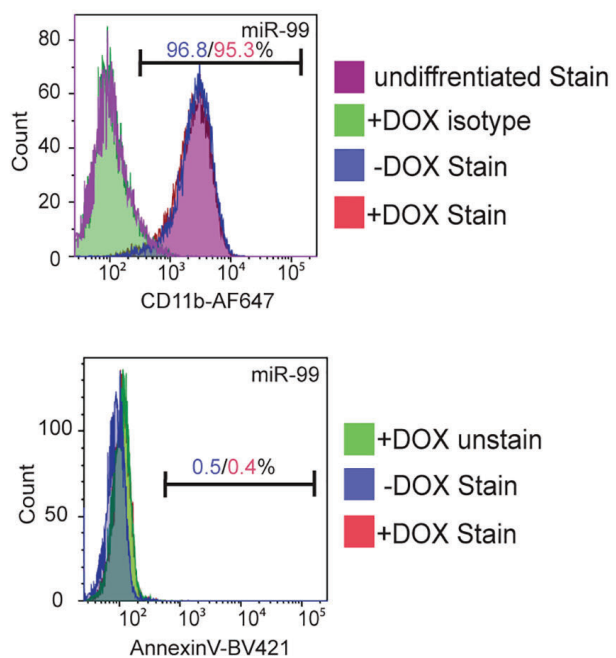

J

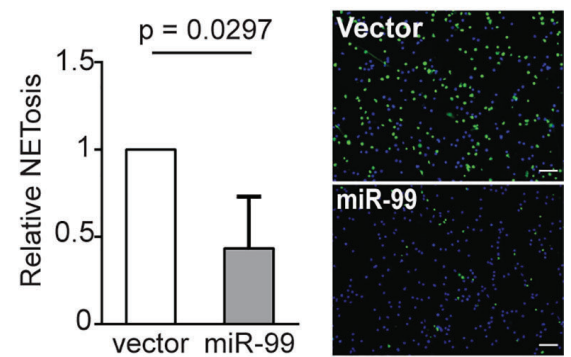

FIGURE 2 | MIR-99 overexpression inhibits chemotaxis of dHL-60 cells. (A) Construct for inducible miRNA expression. The miRNA and a dendra2 reporter (green) are controlled by TRE, Tetracyclin response element (red). The PGK promoter drives constitutive expression of puromycin resistance gene (cyan) and the rtTA, reverse tetracycline-controlled transactivator (red). (B) Quantification of MIR-99, MIR-233, and LET-7E in dHL-60 cell lines with/without induced expression of the vector control or MIR-99. The result from three independent experiments is normalized to U6 expression levels and presented as mean \pm s.d., students' t-test. (C) Cell populations gated for downstream cell profile analysis and transwell quantification. (D) Doxycycline-mediated induction in the vector or miR-99 expressing dHL60 lines. Cells without doxycycline-mediated induction were used as a baseline. Percentage of cells with dendra2 levels above the baseline are shown. (E) Surface CD11b levels in vector or miR-99 expressing dHL-60 cells. Undifferentiated cells or differentiated cells stained with an isotype control were used as a baseline. Percentage of cells with CD11b levels above the baseline with or without doxycycline-mediated induction are shown. (F) Annexin $V$ staining in the total cell population (excluding debris) of vector or miR-99 expressing dHL-60 cells. An unstained live sample is used to determine the baseline. Percentage of cells with AnnexinV levels above the baseline with or without doxycycline-mediated induction are shown. (C-F) One representative experiment of three independent trials is shown. (G-J) (G) Transwell migration of dHL-60 cells with/without induced expression of the vector control or MIR-99 toward fMLP, (H) phagocytosis of BioParticles by dHL60 cells represented as mean FITC, (I) $\mathrm{H}_{2} \mathrm{O}_{2}$ concentration in PMA-induced dHL60 cells, and (J) Quantification of dHL60 undergoing NETosis (left) and

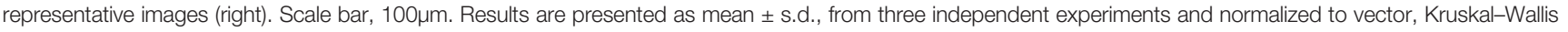
test or Mann-Whitney test.

the ligand-binding domain acts as a dominant-negative by occupying the DNA response elements without activating transcription. We constructed zebrafish lines overexpressing Ror $\alpha$ (1-180aa) in neutrophils, $\operatorname{Tg}($ lyzC:mcherry-2a-rora$d n)^{p u 32}$, together with a control line $\operatorname{Tg}(\operatorname{lyz} \mathrm{C}: \text { mcherry-2a })^{p u 33}$ (Figure 5A). Despite multiple attempts, we could not generate the line overexpressing the full-length rora as a control. Expressing the rora DN did not affect total neutrophil numbers but reduced neutrophil motility in the head mesenchyme (Figures 5B-E and Movie 4). Consistently, Ror $\alpha$ DN significantly reduced neutrophil recruitment to infection or tail amputation sites (Figures 5F-I). The control or the Ror $\alpha$ DN 
A

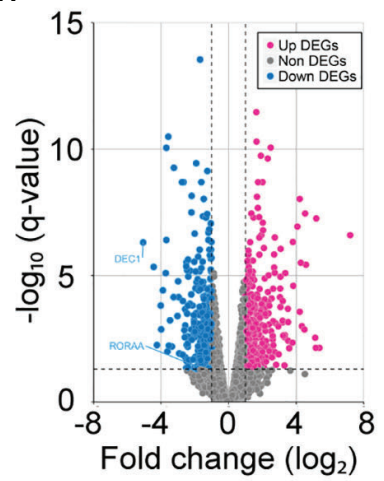

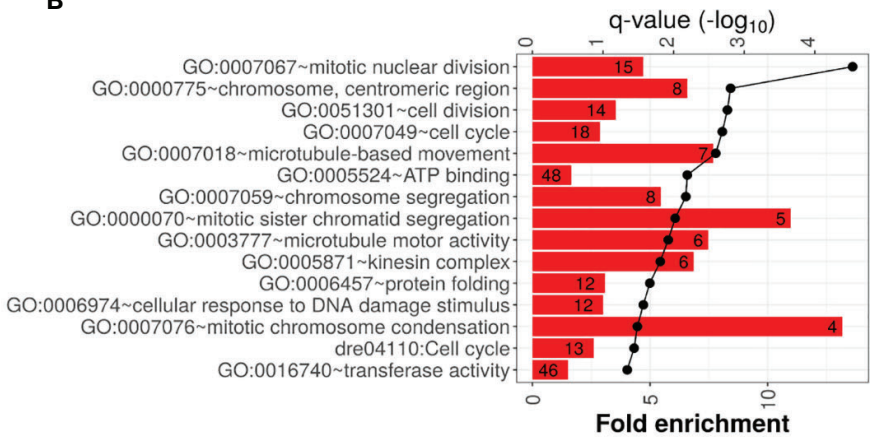

C $\begin{array}{ll}\text { dre-miR-99-1-5p: } & \text { 3'-GUGUUCUAGCCUAGAUGCCCA A-5', } \\ \text { dre-roraa 3'UTR: } & \text { 5'-CGUCGCUCGACAAA UACGGGUU-3' } \\ \text { dre-roraa mut: } & \text { 5'-CGUCGCUCGACAAAAUGCCCA J-3' }\end{array}$

D

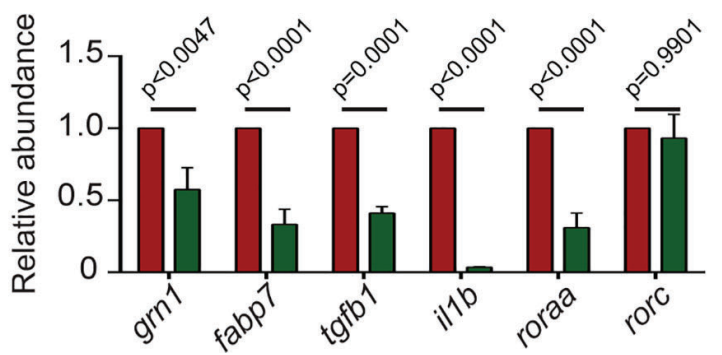

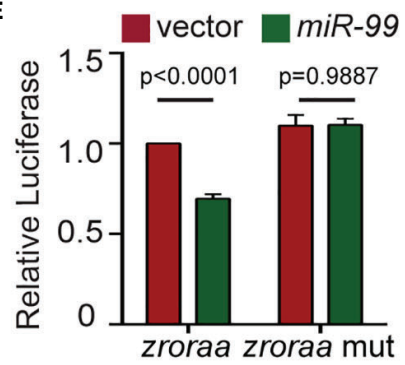

FIGURE 3 | miR-99 overexpression suppresses the expression of roraa in zebrafish neutrophils. (A) Volcano blot of DEGs with significant expression changes in miR-99 expressing neutrophils. Blue: down-regulated differentially expressed genes (DEGs); Magenta: up-regulated DEGs. (B) Significantly altered pathways in the down-regulated DEGs. (C) Alignment of miR-99-1-5p and predicted miR-99 binding sites in zebrafish roraa 3'UTR. (D) Transcript level quantification of predicted downregulated genes in neutrophils sorted from the vector or miR-99 zebrafish line. Results from three independent experiments are normalized to rp/32 and presented as mean. Holm-Sidak test. (E) Suppression of Renilla luciferase expression via binding to zebrafish roraa 3'UTRs by miR-99. Results from three independent experiments are normalized to firefly luciferase and presented as mean \pm s.d., Kruskal-Wallis test.

line was crossed with $\operatorname{Tg}(m p x$ : GFP-UtrCH) to label stable actin in neutrophils (27). In the control neutrophils, stable actin was enriched in the retracing tail. In comparison, in Ror $\alpha$ DN expressing neutrophils, stable actin mislocalized to cell front or the middle of the cell body, indicating disrupted cell polarity (Figure 5J and Movie 5). In addition, we knocked down rora specifically in neutrophils using the CRISPR-Cas9 system recently optimized in our lab where Cas9 expression is restricted in neutrophils and two sgRNAs targeting rora are expressed ubiquitously (49). Consistently, neutrophil migration is significantly inhibited upon rora deletion (Figures $\mathbf{5 K}-\mathbf{N}$ and Movie 6). Furthermore, SR3335 does not further reduce neutrophil speed when the rora gene is disrupted (Figure S2). Taken together, our results indicate that the $\operatorname{ROR} \alpha$ mediated transcription is required for neutrophil polarization and efficient chemotaxis.

\section{ROR $\alpha$ Is Required for Defense Against Bacterial Infection}

Our current finding suggests that miR-99 overexpression or ROR $\alpha$ inhibition suppresses neutrophil chemotaxis. We then investigated whether $\operatorname{ROR} \alpha$ is required for defending against bacterial infection using $P$. aeruginosa (50). Survival of the zebrafish embryos was significantly reduced upon expression of miR-99 or Ror $\alpha$ DN in neutrophils (Figures 6A, B). In line with this observation, inhibiting $\operatorname{ROR} \alpha$, but not $\operatorname{ROR} \gamma$, using small molecules also reduced survival (Figures 6C, D). Taken together, $\mathrm{ROR} \alpha$ plays an essential role in regulating neutrophil activation and the defense against bacterial infection.

\section{Roro Regulates Receptor Signaling Pathways in Zebrafish Neutrophils}

$\mathrm{ROR} \alpha$ is expressed in primary human and zebrafish neutrophils, but not in the human neutrophil model cell line, HL-60, after different differentiation methods (51). Due to the possible offtarget effects of inhibitors and the difficulty of genetically altering primary human neutrophils, we used zebrafish neutrophils overexpressing the Ror $\alpha \mathrm{DN}$ to understand the ROR $\alpha$ regulated gene network., 216 DEGs are down-regulated in zebrafish neutrophils overexpressing Ror $\alpha$ DN compared with the control with a cut off of false discovery rate of 0.05 (Datasets S3, S4). However, the consensus ROR binding motif 


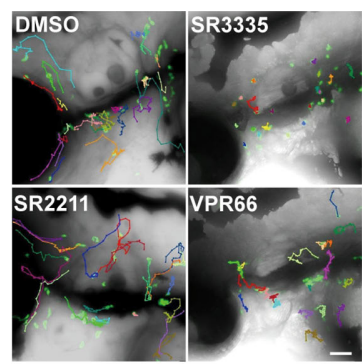

B

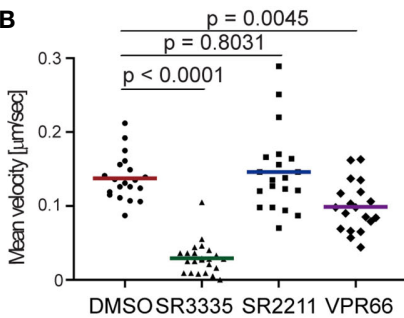

G

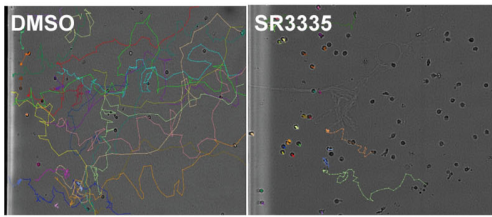

I

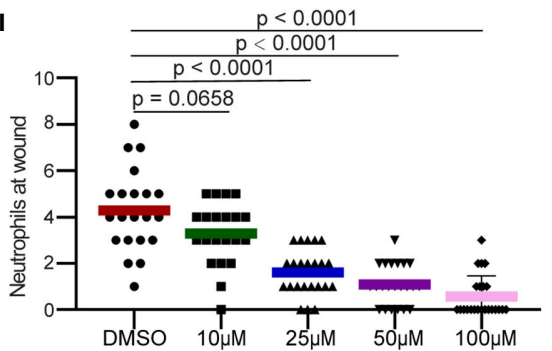

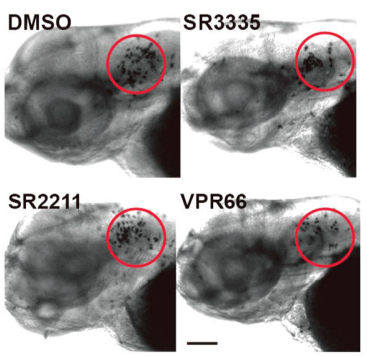

D

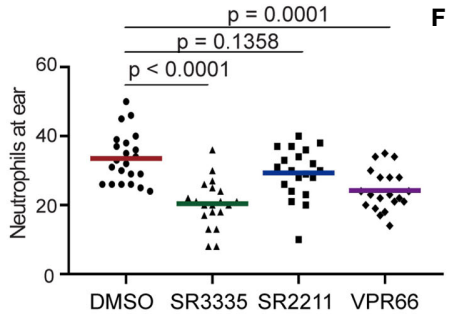

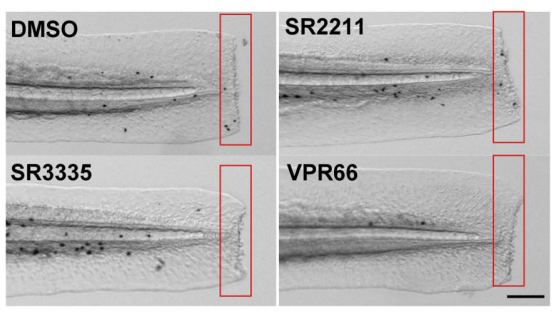

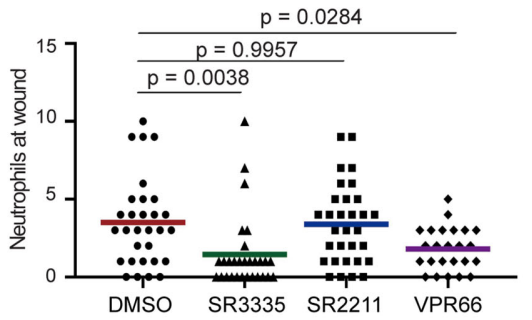

H

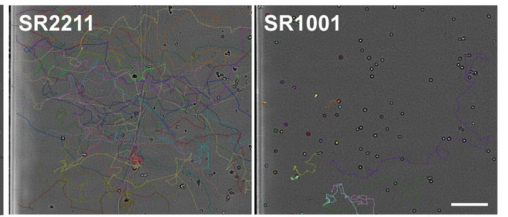

J
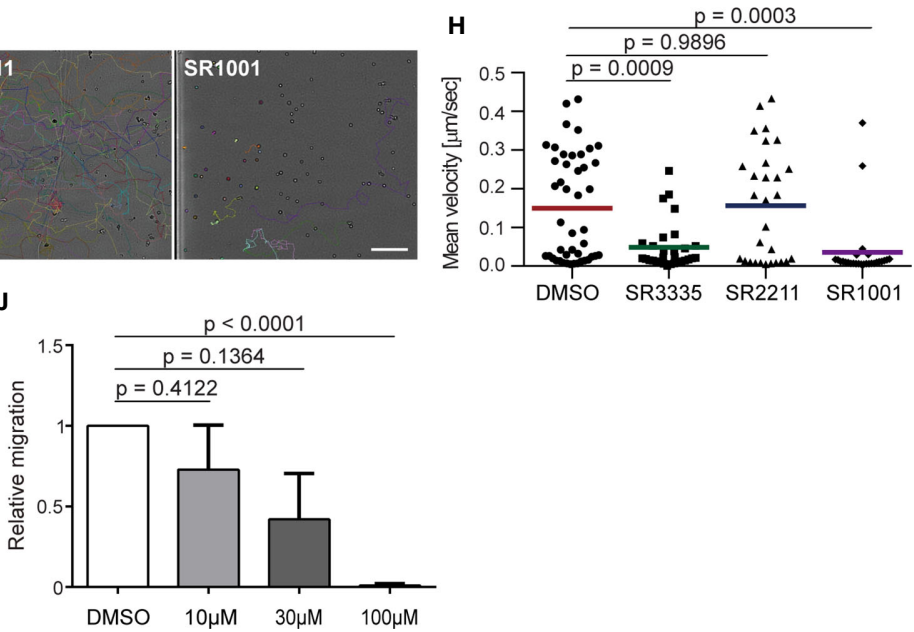

FIGURE 4 | Pharmacological inhibition of Rora reduces neutrophil motility and chemotaxis in zebrafish and humans. (A, B) Tracks and quantification of neutrophil

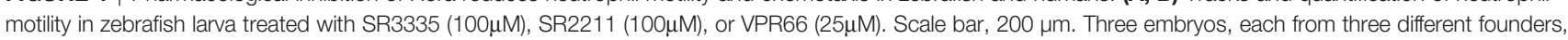
were imaged. Quantification of neutrophils in one representative video is shown, Kruskal-Wallis test. (C, D) Representative images and quantification of neutrophils

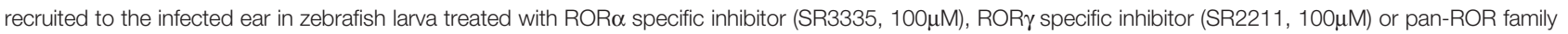

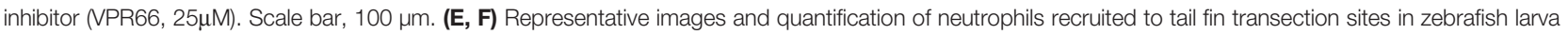
treated with SR3335 $(100 \mu \mathrm{M})$, SR2211 $(100 \mu \mathrm{M})$, or VPR66 $(25 \mu \mathrm{M})$. Scale bar: $500 \mu \mathrm{m}$. (C-F) The result from one representative experiment is shown as mean,

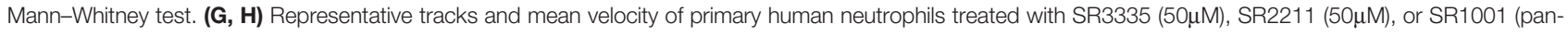
ROR family inhibitor) $(50 \mu \mathrm{M})$ migrating towards fMLP in 3D matrigel. Scale bar, $100 \mu \mathrm{m}$. Representative results for three individual trials are shown. The result is presented as mean, Mann-Whitney test. (I) Neutrophil recruitments after zebrafish tail wounding at different dosages of SR3335 treatment compared to $1 \%$ DMSO treatment, Kruskal-Wallis test. (J) Transwell migration of primary human neutrophils treated with DMSO (0.1\%) or SR3335 at 10,30 , or $100 \mu \mathrm{M}$ toward $100 \mathrm{nM}$ fMLP. Results are presented as mean \pm s.d., from three independent experiments and normalized to DMSO $(0.1 \%)$, Kruskal-Wallis test.

(AxxTxGGTCA) is not enriched in the downregulated DEGs, suggesting direct and indirect transcriptional regulation by ROR $\alpha$. The downregulated DEGs are enriched in cell death, transmembrane signaling receptor activity, and protein phosphorylation pathways (Figures 7A, B). Receptor signaling and protein phosphorylation pathways are involved in cell migration and activation, supporting the biological outcome of ROR $\alpha$ inhibition in neutrophils. We further compared our gene list to a previously published list of $\operatorname{ROR} \alpha$ target genes in monocytic and endothelial cells identified using chromatin immunoprecipitation (ChIP) (52). We converted downregulated DEGs to their human homologs. Ten are putative ROR $\alpha$ target genes in both THP-1 and HUVEC cells, and 13 and 23 are putative ROR $\alpha$ target genes in either one of the cells (Figure 7C) (Supplementary Table 1). Finally, we selected five transcripts that are putative $\mathrm{ROR} \alpha$ target genes to validate this RNA seq result (Figure 7D). As expected, the level of roraa is significantly higher due to overexpression of the DN construct. Rho GTPase 


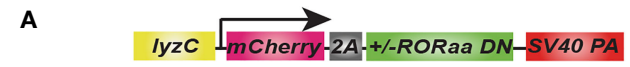

B

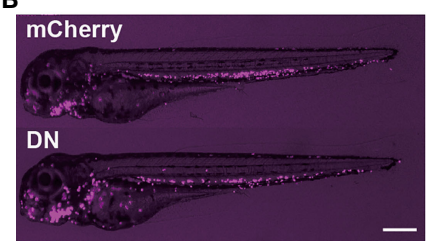

C
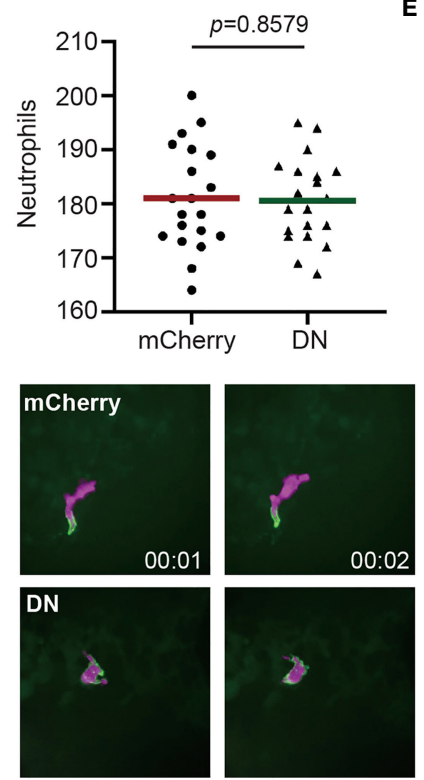

D

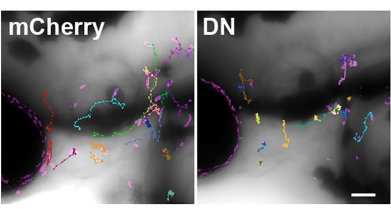

E
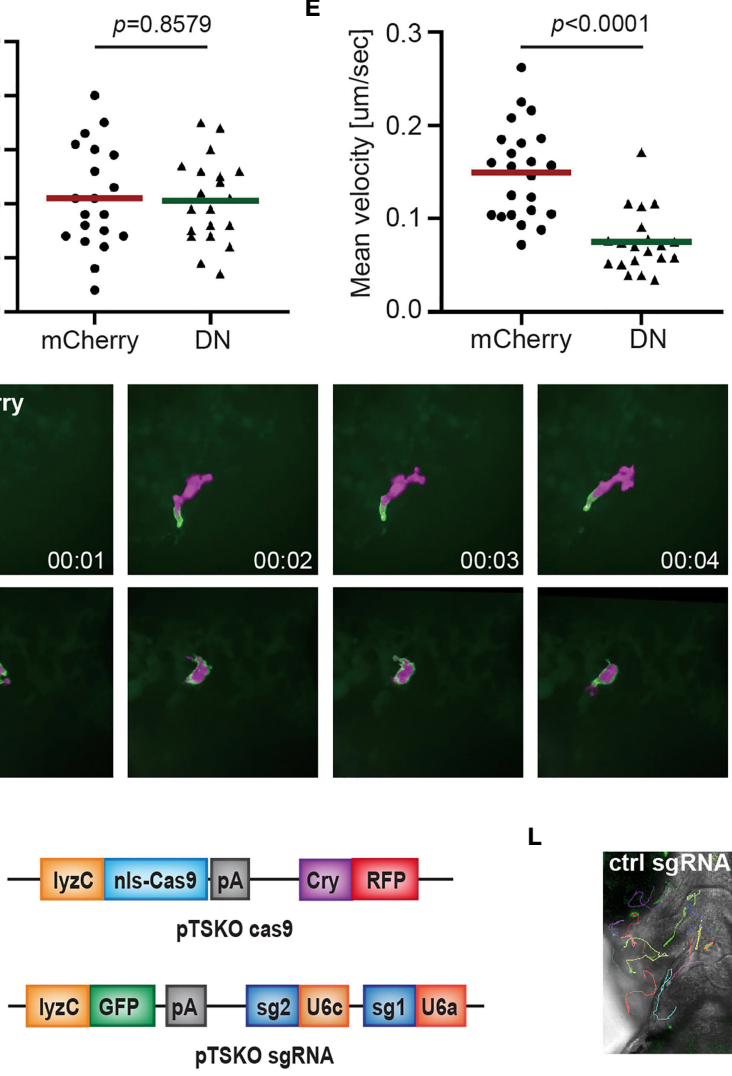

$\mathbf{N}$

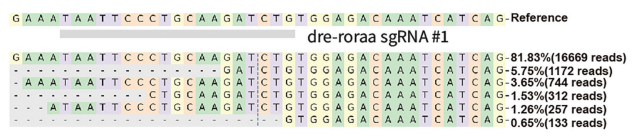

H

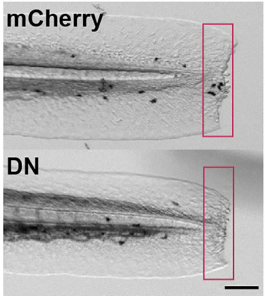

G

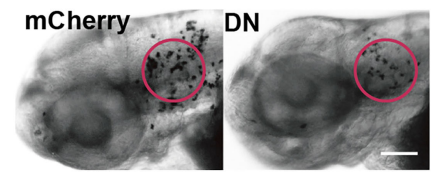

DN
L

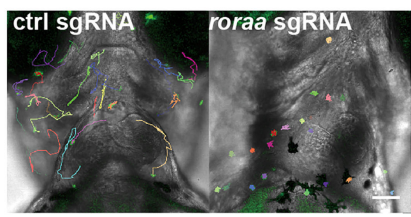

I
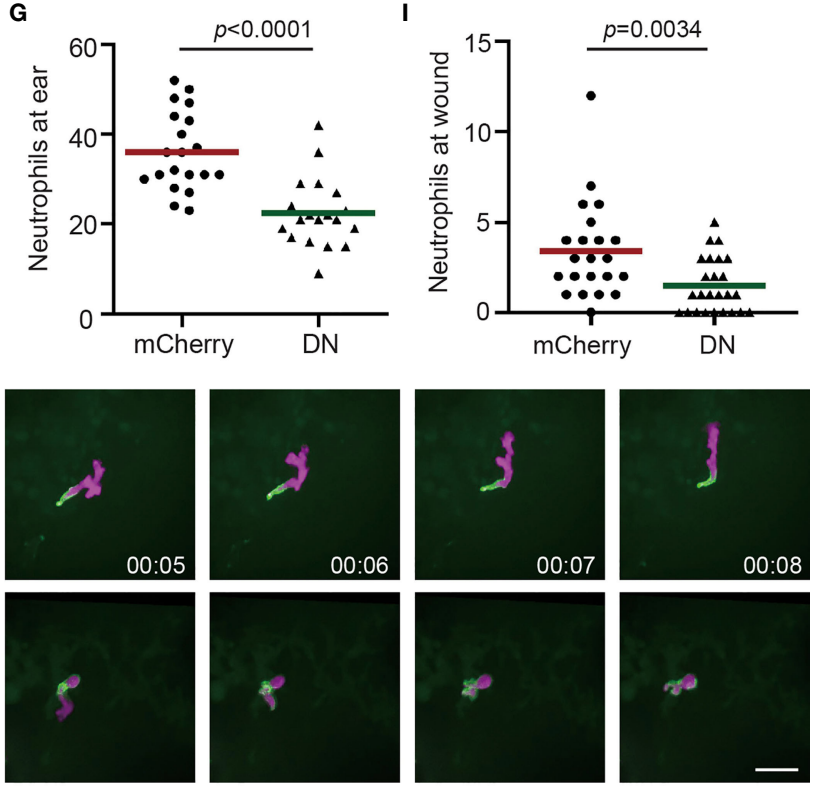

M

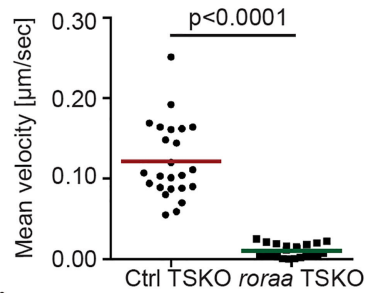

dre-rorag SGRNA H2

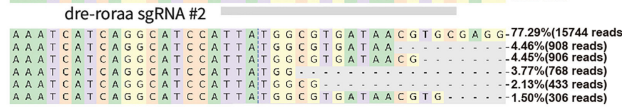

bold Substitutions
$\square$ Insertions

Insertions
Deletions

Predicted cleavage position

FIGURE 5 | Dominant-negative Ror $\alpha$ suppresses neutrophil motility and chemotaxis. (A) Schematic of the construct for neutrophil-specific expression of vector control or ror $\alpha$ dominant-negative (DN). (B, C) Representative images and quantification of total neutrophil numbers in vector or ror $\alpha$ DN zebrafish line. Scale bar: $500 \mu \mathrm{m}$. (D, E) Representative images and velocity of random neutrophil migration in vector or ror $\alpha$ DN zebrafish line. Scale bar, $100 \mu \mathrm{m}$. Three embryos each from three different founders were imaged, and quantification of neutrophils in one representative video is shown, Kruskal-Wallis test. (F, G) Representative images and quantification of neutrophils recruited to the infected ear in vector or ror $\alpha$ DN zebrafish line. Scale bar, $100 \mu \mathrm{m}$. (H, I) Representative images and quantification of neutrophil recruitment to the tailfin transection sites in vector or ror $\alpha$ DN zebrafish line. Scale bar, $200 \mu m$. The result is presented as mean, Mann-Whitney test. (J) Simultaneous imaging of utrophin-GFP distribution in neutrophils expressing either mCherry alone or with Rora DN. Data are representative of more than three separate time-lapse videos. Scale Bar, 50 Mm. (B, C, F-I) Representative results for three individual trials are shown. (K) Schematics of the plasmid constructs for neutrophil-specific Cas9 expression (upper construct) and for ubiquitous expression of sgRNAs with a neutrophil-specific expression of GFP (lower construct). (L, M) Representative images (L) and quantification (M) of neutrophil motility at head region of 3dpf larvae from Tg(LyzC: Cas9, Cry: GRFP, U6a/C: rora guides, LyzC: GFP) fish. Student's t-test. Scale bar, $100 \mu \mathrm{m}$. (N) Deep sequencing of the rora loci targeted by guide RNAs in (K). The sequences on the top are wild-type sequences, and the five most frequent types of mutations are shown. Point mutations, deletions, and insertions are all observed. 


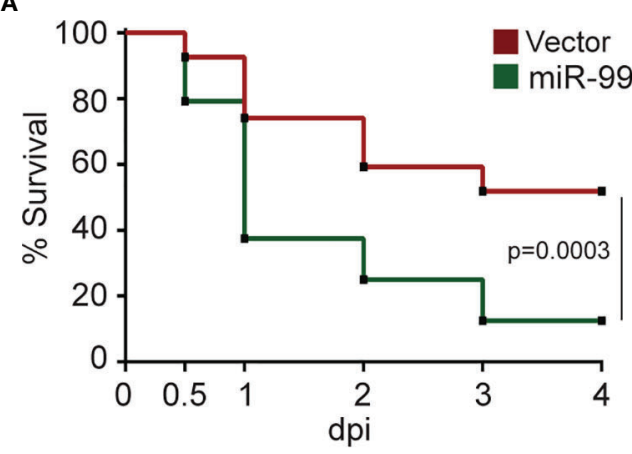

C

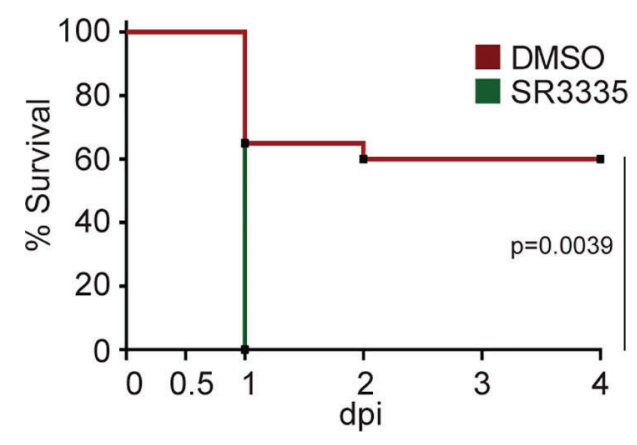

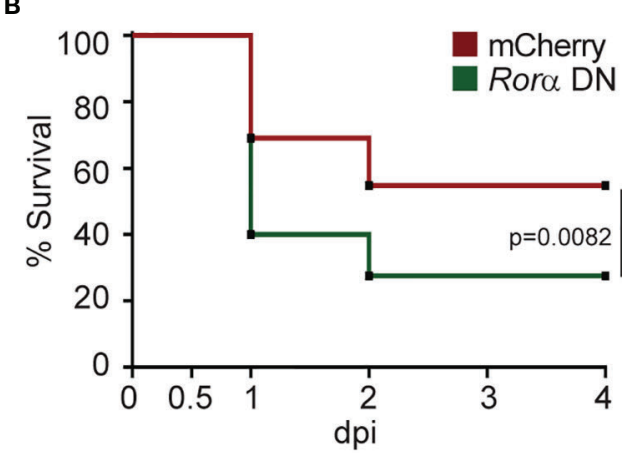

D

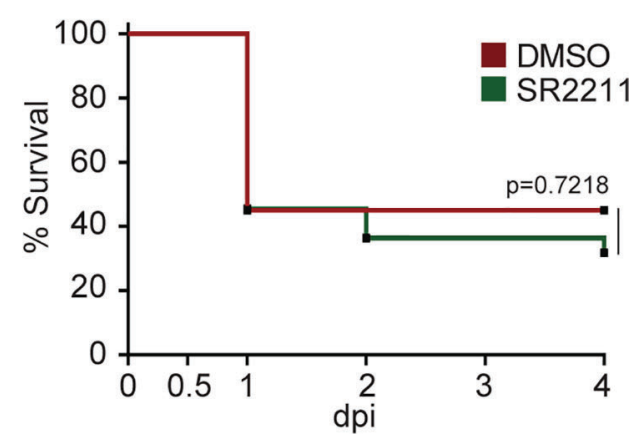

FIGURE 6 | Ror $\alpha$ in neutrophils protects zebrafish against Pseudomonas aeruginosa infection. (A) Survival curve of 3 dpf larvae from the vector or miR-99 line after i.v. infection with Pseudomonas aeruginosa (PAK). (B) Survival curve of 3 dpf larvae from the mcherry or roraa DN line larvae after i.v. infection with PAK. (C) Survival

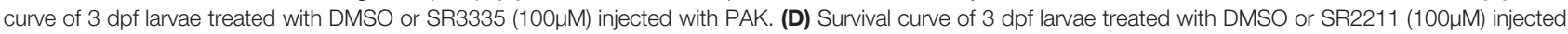
with PAK. One representative experiment of three independent biological repeats ( $n=20$ each group) is shown. The result is analyzed with Gehan-BreslowWilcoxon test.

Activating Protein 17 (Arhgap17b) is a signaling molecule regulating Cdc42 and Racl (53). Protein Tyrosine Kinase 2 Beta (Ptk2ba) regulates protein tyrosine kinase activity and is implicated in human neutrophil migration (54) and host defense response to bacterial infection (55). MHC Class I Polypeptide-
Related Sequence B (Mhclzba) is essential in self-antigen presentation under stressed conditions recognized by innatelike lymphocytes (56). Calcium Voltage-Gated Channel Auxiliary Subunit Gamma 3 (Cacng3b) is among the most significantly altered DEGs. It is a voltage-dependent calcium
A

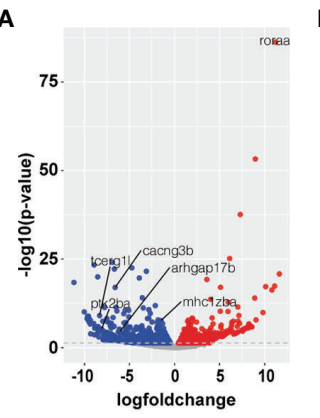

B

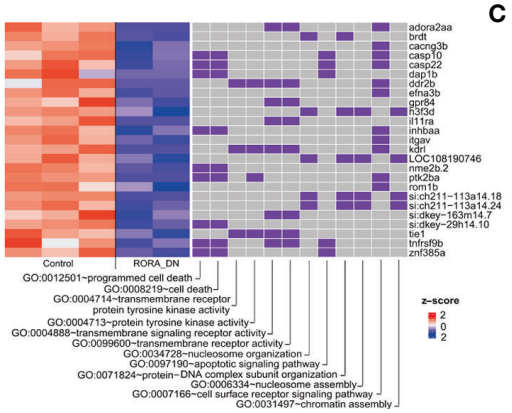

C

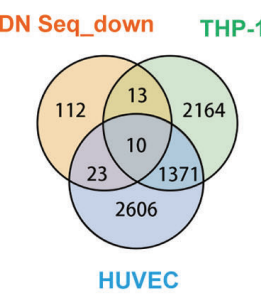

D

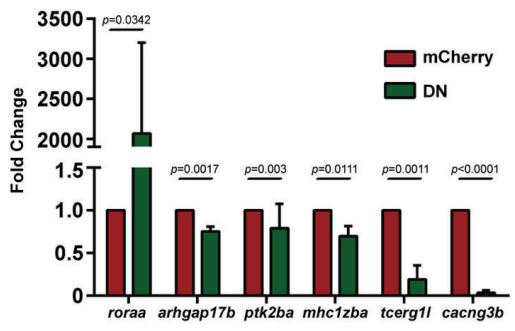

FIGURE 7 | RNA-Seq reveals the direction of ror $\alpha$ possible downstream genes in neutrophil migration. (A) Volcano blot of DEGs with significant expression changes in ror $\alpha$ DN neutrophils. Cyan: down-regulated differentially expressed genes (DEGs); Magenta: up-regulated DEGs. (B) Significant gene function enrichment of human homologs of genes in the down-regulated DEGs. The heat map indicates the log2 fold change of the selected DEGs. The relevance of individual DEGs in each pathway is indicated with purple. (C) Venn diagram showing human homologs of genes in the down-regulated DEGs overlapping with putative ROR $\alpha$ target genes in THP-1 or HUVEC cells. (D) Transcript level validation of downregulated genes in neutrophils sorted from ror $\alpha$ DN line zebrafish. Results from three independent experiments are normalized to rp/32 and presented as mean \pm s.d., students' t-test. 
channel subunit on the plasma membrane, regulating the receptor activity (57). Another most significantly changed DEG we validated is the transcription elongation regulator one like (Tcerg11). Together, the validated downregulation of these molecules provides a molecular understanding of how ROR $\alpha$ dependent gene expression is required for proper neutrophil migration and activation.

\section{DISCUSSION}

In the present study, we characterized miR-99 as a critical regulator of neutrophil migration and chemotaxis. miR-99 hinders neutrophil motility by downregulating genes enriched in zebrafish receptor activity pathways and directly targeting roraa. We further demonstrate that the transcriptional regulation activity of $\operatorname{ROR} \alpha$ is critical for neutrophil migration and defense against a bacterial pathogen.

MIR-99 has multiple regulatory roles in various biological processes, especially hematopoietic and leukemic stem cell differentiation and function (58). The miR-99/100 125 microRNA cluster block of the transforming growth factor $\beta$ (TGF $\beta$ ) pathway enhances Wnt signaling to promote the survival and expansion of hematopoietic stem cells megakaryocytic differentiation (59). miR-99 is highly expressed in hematopoietic stem cells (HSCs) and acute myeloid leukemia stem cells (LSCs) and promotes their self-renewal and leukemia-initiating activity through targeting Hoxa1 (60). Outside the blood system, miR-99 is a tumor suppressor. miR-99 suppresses prostate cancer cell proliferation by suppressing the expression of prostate-specific antigen (61). It also stops expanding the human cervical cancer cell line, HeLa, targeting Tribbles 2 (62). MiR-99 suppresses FGFR3 expression in lung cancer and Erk1/2 and Akt, reducing cell growth and metastasis (63). In addition, miR-99 is transiently induced after radiation, reducing the efficiency of DNA repair by suppressing SNF2H expression (64). miR-99 family is highly downregulated in physiological but up-regulated during pathological hypertrophy (65). No prior studies have investigated the role of miR-99 in neutrophils. Therefore, our discovery that miR-99 is a suppressor of cell migration in leukocytes expands the biological functions of miR-99 outside cancer biology.

We identified that retinoid acid receptor-related orphan receptor $\alpha(\mathrm{ROR} \alpha)$ is a direct target of miR-99. Dominantacting $\operatorname{ROR} \alpha$ mutations are associated with autism and cerebellar ataxia in humans (66). ROR $\alpha$ polymorphisms correlate with human diseases such as breast cancer (42) and asthma (43). ROR $\alpha$ gene expression is downregulated in the blood of multiple sclerosis patients (41). Both pro-inflammatory $(67,68)$ and anti-inflammatory $(69,70)$ roles of $\operatorname{ROR} \alpha$ are observed in different cell types. ROR $\alpha$ deficient mast cells and macrophages produce increased amounts of TNF-alpha and IL-6 upon activation (69). Ectopic expression of ROR alpha1 inhibits TNF alpha-induced IL-6, IL-8, and COX-2 expression in human primary smooth-muscle cells, through the induction of a significant inhibitory protein I kappa B alpha (70). In contrast, $\mathrm{ROR} \alpha$ suppressed the expression of suppressors of cytokine signaling 3 (SOCS3), a negative regulator of inflammation, and deletion of $\operatorname{ROR} \alpha$ protects against oxygen-induced proliferative retinopathy in a murine model (67). ROR $\alpha$ stimulates inflammation in adipose tissue by enhancing the expression of ER stress response genes and inflammatory cytokines (68). In the intestinal epithelial cell, ROR $\alpha$ attenuates NF-kB activity and inhibits excessive inflammation in a mouse colitis model (71). In human neutrophils, endotoxin administration downregulates the expression of ROR $\alpha$ (72). However, it is not previously known if $\mathrm{ROR} \alpha$ plays a role in regulating neutrophil function. We used both inhibitor (affecting the whole embryo) and transgenic lines involving $\mathrm{ROR} \alpha$ only in neutrophils. Our results demonstrated that $\operatorname{ROR} \alpha$ in neutrophils are essential for migration and antibacterial defense, but we cannot rule out that $\mathrm{ROR} \alpha$ in other cells also contribute to the antibacterial defense.

Although we characterized the gene expression network regulated by $\operatorname{ROR} \alpha$ in zebrafish neutrophils and provided the molecular understanding that $\mathrm{ROR} \alpha$ regulates receptor activation and downstream signaling pathways. The limitation of the current study is that the results are yet to be confirmed in human neutrophils. The most surprising result is that chemotaxis of primary human neutrophils is reduced after only $1 \mathrm{hr}$ of ROR $\alpha$ inhibitor treatment, given that human neutrophils possess limited transcriptional activity and do not require transcription for chemotaxis $(47,48)$. One possibility is that SR3335 has off-target effects, and the phenotype is not related to ROR $\alpha$. Another scenario is that $\operatorname{ROR} \alpha$ activity needs to be maintained to support neutrophil chemotaxis. The previous work identified limited transcriptional activity (48) used differentiated HL-60 cells, which does not express ROR $\alpha$. Therefore, the conclusion should be revisited in primary human neutrophils. However, due to the short half-life of primary human neutrophils, metabolic labeling to identify nascent mRNA in this cell type is challenging. A list of active transcripts in zebrafish neutrophils is also obtained by isolating associated ribosomal mRNAs (73). Although this method can identify genes responsive to tissue damage, due to the high background in the IP (false positives), this result cannot be used to infer a reliable, complete list of active transcripts. Future studies will be needed to carefully set up the assays to get to the functional transcripts in human neutrophils and identify those regulated by ROR $\alpha$. In addition, neutrophils display rhythmical behavior mediated by core clock genes in mice (74) and zebrafish (75). In the future, it is also interesting to determine how ROR $\alpha$ regulates the rhythmical behavior of neutrophils.

In summary, our data indicated that the miR-99/ROR $\alpha$ axis functions as a novel modulator in the acute stage of neutrophil recruitment and is potentially a novel target in preventing and treating acute inflammatory diseases and infections. There are neutrophil-focused drug candidates in clinical trials, including CXCR2 blockades, neutrophil elastase inhibitors, and C5a receptor antagonists. $\mathrm{ROR} \alpha$ blockades may turn out to be efficient immune suppressants. Nanotechnology-based drug delivery (76) has shown promise in blocking the airway and systemic inflammation (77). Neutrophils are loaded with therapeutic cargos ex vivo and reintroduced to animals to treat tumors (78). Developing techniques allowing neutrophil-specific 
delivery of microRNA mimics or ROR $\alpha$ inhibitors in vivo will further facilitate the translation of our results into clinics.

\section{DATA AVAILABILITY STATEMENT}

The RNA-seq raw and processed data are submitted to the Gene Expression Omnibus (https://www.ncbi.nlm.nih.gov/geo/query/acc. cgi?acc=GSE144873; https://www.ncbi.nlm.nih.gov/geo/query/acc. cgi? acc $=$ GSE171501). Plasmids are available on Addgene.

\section{ETHICS STATEMENT}

The studies involving human participants were reviewed and approved by Purdue's Institutional Review Board. The patients/ participants provided their written informed consent to participate in this study. The animal study was reviewed and approved by IACUC.

\section{AUTHOR CONTRIBUTIONS}

$\mathrm{AH}, \mathrm{TW}, \mathrm{RS}, \mathrm{SM}, \mathrm{CS}, \mathrm{JuW}$ and QD designed research and wrote the manuscript. AH, TW, RS, WZ, JiW, ZC, ST, and ZC

\section{REFERENCES}

1. Mocsai A. Diverse Novel Functions of Neutrophils in Immunity, Inflammation, and Beyond. J Exp Med (2013) 210:1283-99. doi: 10.1084/ jem. 20122220

2. Kolaczkowska E, Kubes P. Neutrophil Recruitment and Function in Health and Inflammation. Nat Rev Immunol (2013) 13:159-75. doi: 10.1038/nri3399

3. Peiseler M, Kubes P. More Friend Than Foe: The Emerging Role of Neutrophils in Tissue Repair. J Clin Invest (2019) 129:2629-39. doi: $10.1172 /$ JCI124616

4. Soehnlein O, Steffens S, Hidalgo A, Weber C. Neutrophils as Protagonists and Targets in Chronic Inflammation. Nat Rev Immunol (2017) 17:248-61. doi: 10.1038/nri.2017.10

5. Nemeth T, Sperandio M, Mocsai A. Neutrophils as Emerging Therapeutic Targets. Nat Rev Drug Discov (2020) 19:253-75. doi: 10.1038/s41573-0190054-z

6. Deng Q, Huttenlocher A. Leukocyte Migration From a Fish Eye's View. J Cell Sci (2012) 125:3949-56. doi: 10.1242/jcs.093633

7. Novoa B, Figueras A. Zebrafish: Model for the Study of Inflammation and the Innate Immune Response to Infectious Diseases. Adv Exp Med Biol (2012) 946:253-75. doi: 10.1007/978-1-4614-0106-3_15

8. Fabian MR, Sonenberg N. The Mechanics of miRNA-Mediated Gene Silencing: A Look Under the Hood of miRISC. Nat Struct Mol Biol (2012) 19:586-93. doi: 10.1038/nsmb.2296

9. Broderick JA, Zamore PD. MicroRNA Therapeutics. Gene Ther (2011) 18:1104-10. doi: 10.1038/gt.2011.50

10. Chakraborty C, Sharma AR, Sharma G, Doss CGP, Lee SS. Therapeutic miRNA and siRNA: Moving From Bench to Clinic as Next Generation Medicine. Mol Ther Nucleic Acids (2017) 8:132-43. doi: 10.1016/ j.omtn.2017.06.005

11. Hsu AY, Wang D, Liu S, Lu J, Syahirah R, Bennin DA, et al. Phenotypical microRNA Screen Reveals a Noncanonical Role of CDK2 in Regulating Neutrophil Migration. Proc Natl Acad Sci USA (2019) 116:18561-70. doi: 10.1073/pnas.1905221116

12. Lau P, Fitzsimmons RL, Pearen MA, Watt MJ, Muscat GE. Homozygous Staggerer $(\mathrm{Sg} / \mathrm{Sg})$ Mice Display Improved Insulin Sensitivity and Enhanced performed the experiments. AH, TW, RS, KL, and SL analyzed data. All authors read and approved the manuscript.

\section{FUNDING}

The work was supported by research funding from the National Institutes of Health (R35GM119787 to QD), and (P30CA023168 to Purdue Center for Cancer Research) for shared resources. The author(s) acknowledge the use of the facilities of the Bindley Bioscience Center, a core facility of the NIH-funded Indiana Clinical and Translational Sciences Institute. Bioinformatics analysis was conducted by the Collaborative Core for Cancer Bioinformatics $\left(\mathrm{C}^{3} \mathrm{~B}\right)$ shared by the Indiana University Simon Cancer Center (P30CA082709) and the Purdue University Center for Cancer Research with support from the Walther Cancer Foundation.

\section{SUPPLEMENTARY MATERIAL}

The Supplementary Material for this article can be found online at: https://www.frontiersin.org/articles/10.3389/fimmu.2022.756034/ full\#supplementary-material

Glucose Uptake in Skeletal Muscle. Diabetologia (2011) 54:1169-80. doi: 10.1007/s00125-011-2046-3

13. Kang HS, Okamoto K, Takeda Y, Beak JY, Gerrish K, Bortner CD, et al. Transcriptional Profiling Reveals a Role for RORalpha in Regulating Gene Expression in Obesity-Associated Inflammation and Hepatic Steatosis. Physiol Genomics (2011) 43:818-28. doi: 10.1152/physiolgenomics.00206.2010

14. Sato TK, Panda S, Miraglia LJ, Reyes TM, Rudic RD, McNamara P, et al. A Functional Genomics Strategy Reveals Rora as a Component of the Mammalian Circadian Clock. Neuron (2004) 43:527-37. doi: 10.1016/ j.neuron.2004.07.018

15. Ferreira ACF, Szeto ACH, Heycock MWD, Clark PA, Walker JA, Crisp A, et al. RORalpha Is a Critical Checkpoint for T Cell and ILC2 Commitment in the Embryonic Thymus. Nat Immunol (2021) 22:166-78. doi: 10.1038/ s41590-020-00833-w

16. Rajput C, Cui T, Han M, Lei J, Hinde JL, Wu Q, et al. RORalpha-Dependent Type 2 Innate Lymphoid Cells Are Required and Sufficient for Mucous Metaplasia in Immature Mice. Am J Physiol Lung Cell Mol Physiol (2017) 312:L983-93. doi: 10.1152/ajplung.00368.2016

17. Malhotra N, Leyva-Castillo JM, Jadhav U, Barreiro O, Kam C, O'Neill NK, et al. RORalpha-Expressing $\mathrm{T}$ Regulatory Cells Restrain Allergic Skin Inflammation. Sci Immunol (2018) 3(21):eaao6923. doi: 10.1126/ sciimmunol.aao6923

18. Wang R, Campbell S, Amir M, Mosure SA, Bassette MA, Eliason A, et al. Genetic and Pharmacological Inhibition of the Nuclear Receptor RORalpha Regulates TH17 Driven Inflammatory Disorders. Nat Commun (2021) 12:76. doi: 10.1038/s41467-020-20385-9

19. Yang XXO, Pappu BP, Nurieva R, Akimzhanov A, Kang HS, Chung Y, et al. T Helper 17 Lineage Differentiation Is Programmed by Orphan Nuclear Receptors ROR Alpha and ROR Gamma. Immunity (2008) 28:29-39. doi: 10.1016/j.immuni.2007.11.016

20. Chi X, Jin W, Bai X, Zhao X, Shao J, Li J, et al. RORalpha Is Critical for Mtorc1 Activity in T Cell-Mediated Colitis. Cell Rep (2021) 36:109682. doi: 10.1016/ j.celrep.2021.109682

21. Haim-Vilmovsky L, Henriksson J, Walker JA, Miao Z, Natan E, Kar G, et al. Mapping Rora Expression in Resting and Activated CD4+ T Cells. PloS One (2021) 16:e0251233. doi: 10.1371/journal.pone.0251233 
22. Song J, Song H, Wei H, Sun R, Tian Z, Peng H. Requirement of RORalpha for Maintenance and Anti-Tumor Immunity of Liver-Resident Natural Killer Cells/ILC1s. Hepatology (2021) 00:1-13. doi: 10.1002/hep.32147

23. Hams E, Roberts J, Bermingham R, Fallon PG. Functions for Retinoic AcidRelated Orphan Receptor Alpha (ROR Alpha) in the Activation of Macrophages During Lipopolysaccharide-Induced Septic Shock. Front Immunol (2021) 12. doi: 10.3389/fimmu.2021.647329

24. Hams E, Roberts J, Bermingham R, Hogan AE, O'Shea D, O'Neill L, et al. Role for Retinoic Acid-Related Orphan Receptor Alpha (ROR Alpha) Expressing Macrophages in Diet-Induced Obesity. Front Immunol (2020) 11. doi: 10.3389/fimmu.2020.01966

25. Lo BC, Gold MJ, Hughes MR, Antignano F, Valdez Y, Zaph C, et al. The Orphan Nuclear Receptor RORalpha and Group 3 Innate Lymphoid Cells Drive Fibrosis in a Mouse Model of Crohn's Disease. Sci Immunol (2016) 1: eaaf8864. doi: 10.1126/sciimmunol.aaf8864

26. Farr JN, Weivoda MM, Nicks KM, Fraser DG, Negley BA, Onken JL, et al. Osteoprotection Through the Deletion of the Transcription Factor Ror in Mice. J Bone Miner Res (2018) 33:720-31. doi: 10.1002/jbmr.3351

27. Deng Q, Yoo SK, Cavnar PJ, Green JM, Huttenlocher A. Dual Roles for Rac2 in Neutrophil Motility and Active Retention in Zebrafish Hematopoietic Tissue. Dev Cell (2011) 21:735-45. doi: 10.1016/j.devcel.2011.07.013

28. Dobin A, Davis CA, Schlesinger F, Drenkow J, Zaleski C, Jha S, et al. STAR: Ultrafast Universal RNA-Seq Aligner. Bioinformatics (2013) 29:15-21. doi: 10.1093/bioinformatics/bts635

29. Liao Y, Smyth GK, Shi W. Featurecounts: An Efficient General Purpose Program for Assigning Sequence Reads to Genomic Features. Bioinformatics (2014) 30:923-30. doi: 10.1093/bioinformatics/btt656

30. Huang da W, Sherman BT, Lempicki RA. Systematic and Integrative Analysis of Large Gene Lists Using DAVID Bioinformatics Resources. Nat Protoc (2009) 4:44-57. doi: 10.1038/nprot.2008.211

31. Hsu AY, Wang DC, Gurol T, Zhou WQ, Zhu XG, Lu HY, et al. Overexpression of microRNA-722 Fine-Tunes Neutrophilic Inflammation by Inhibiting Rac2 in Zebrafish. Dis Models Mech (2017) 10:1323-32. doi: $10.1242 / \mathrm{dmm} .030791$

32. Cavnar PJ, Berthier E, Beebe DJ, Huttenlocher A. Hax1 Regulates Neutrophil Adhesion and Motility Through RhoA. J Cell Biol (2011) 193:465-73. doi: $10.1083 /$ jcb. 201010143

33. Hsu AY, Liu S, Syahirah R, Brasseale KA, Wan J, Deng Q. Inducible Overexpression of Zebrafish microRNA-722 Suppresses Chemotaxis of Human Neutrophil Like Cells. Mol Immunol (2019) 112:206-14. doi: 10.1016/j.molimm.2019.06.001

34. Hall C, Flores MV, Storm T, Crosier K, Crosier P. The Zebrafish Lysozyme C Promoter Drives Myeloid-Specific Expression in Transgenic Fish. BMC Dev Biol (2007) 7:42. doi: 10.1186/1471-213X-7-42

35. Henry KM, Loynes CA, Whyte MKB, Renshaw SA. Zebrafish as a Model for the Study of Neutrophil Biology. J Leukoc Biol (2013) 94:633-42. doi: 10.1189/ jlb.1112594

36. Jacob C, Leport M, Szilagyi C, Allen JM, Berttand C, Lagente V. DMSOTreated HL60 Cells: A Model of Neutrophil-Like Cells Mainly Expressing PDE4B Subtype. Int Immunopharmacol (2002) 2:1647-56. doi: 10.1016/ S1567-5769(02)00141-8

37. He L, Hannon GJ. Micrornas: Small RNAs With a Big Role in Gene Regulation. Nat Rev Genet (2004) 5:522-31. doi: 10.1038/nrg1379

38. Kojetin DJ, Burris TP. REV-ERB and ROR Nuclear Receptors as Drug Targets. Nat Rev Drug Discov (2014) 13:197-216. doi: 10.1038/nrd4100

39. Zhang Y, Luo XY, Wu DH, Xu Y. ROR Nuclear Receptors: Structures, Related Diseases, and Drug Discovery (Vol 36, Pg 71, 2015). Acta Pharmacol Sin (2015) 36(1):71-87. doi: 10.1038/aps.2014.120

40. Roforth MM, Liu G, Khosla S, Monroe DG. Examination of Nuclear Receptor Expression in Osteoblasts Reveals Ror Ss as an Important Regulator of Osteogenesis. J Bone Miner Res (2012) 27:891-901. doi: 10.1002/jbmr.1502

41. Sayad A, Salmani T, Hemmesi MK, Ganji M, Ghafouri-Fard S, Hatami M, et al. Down-Regulation of RORA Gene Expression in the Blood of Multiple Sclerosis Patients. Hum Antibodies (2018) 26:219-24. doi: 10.3233/HAB-180341

42. Taheri M, Omrani MD, Noroozi R, Ghafouri-Fard S, Sayad A. Retinoic AcidRelated Orphan Receptor Alpha (RORA) Variants and Risk of Breast Cancer. Breast Dis (2017) 37:21-5. doi: 10.3233/BD-160248
43. Cai X, Lin M, Cao S, Liu Y, Lin N. The Association of RAR-Related Orphan Receptor A (RORA) Gene Polymorphisms With the Risk of Asthma. Ann Hum Genet (2018) 82:158-64. doi: 10.1111/ahg.12238

44. Evans RM. The Steroid and Thyroid Hormone Receptor Superfamily. Science (1988) 240:889-95. doi: 10.1126/science.3283939

45. Wang Y, Kumar N, Solt LA, Richardson TI, Helvering LM, Crumbley C, et al. Modulation of Retinoic Acid Receptor-Related Orphan Receptor Alpha and Gamma Activity by 7-Oxygenated Sterol Ligands. J Biol Chem (2010) 285:5013-25. doi: 10.1074/jbc.M109.080614

46. Solt LA, Burris TP. Action of RORs and Their Ligands in (Patho)Physiology Trends Endocrinol Metab (2012) 23:619-27. doi: 10.1016/j.tem.2012.05.012

47. Tecchio C, Micheletti A, Cassatella MA. Neutrophil-Derived Cytokines: Facts Beyond Expression. Front Immunol (2014) 5:508. doi: 10.3389/ fimmu.2014.00508

48. Yost CC, Denis MM, Lindemann S, Rubner FJ, Marathe GK, Buerke M, et al. Activated Polymorphonuclear Leukocytes Rapidly Synthesize Retinoic Acid Receptor-Alpha: A Mechanism for Translational Control of Transcriptional Events. J Exp Med (2004) 200:671-80. doi: 10.1084/jem.20040224

49. Wang Y, Hsu AY, Walton EM, Park SJ, Syahirah R, Wang T, et al. A Robust and Flexible CRISPR/Cas9-Based System for Neutrophil-Specific Gene Inactivation in Zebrafish. J Cell Sci (2021) 134(8):jcs258574. doi: 10.1242/ jcs. 258574

50. Brannon MK, Davis JM, Mathias JR, Hall CJ, Emerson JC, Crosier PS, et al. Pseudomonas Aeruginosa Type III Secretion System Interacts With Phagocytes to Modulate Systemic Infection of Zebrafish Embryos. Cell Microbiol (2009) 11:755-68. doi: 10.1111/j.1462-5822.2009.01288.x

51. Rincon E, Rocha-Gregg BL, Collins SR. A Map of Gene Expression in Neutrophil-Like Cell Lines. BMC Genomics (2018) 19:573. doi: 10.1186/ s12864-018-4957-6

52. Gulec C, Coban N, Ozsait-Selcuk B, Sirma-Ekmekci S, Yildirim O, ErginelUnaltuna N. Identification of Potential Target Genes of ROR-Alpha in THP1 and HUVEC Cell Lines. Exp Cell Res (2017) 353:6-15. doi: 10.1016/ j.yexcr.2017.02.028

53. Richnau N, Aspenstrom P. Rich, a Rho GTPase-Activating Protein DomainContaining Protein Involved in Signaling by Cdc42 and Rac1. J Biol Chem (2001) 276:35060-70. doi: 10.1074/jbc.M103540200

54. Di Cioccio V, Strippoli R, Bizzarri C, Troiani G, Cervellera MN, Gloaguen I, et al. Key Role of Proline-Rich Tyrosine Kinase 2 in Interleukin-8 (CXCL8/IL8)-Mediated Human Neutrophil Chemotaxis. Immunology (2004) 111:40715. doi: 10.1111/j.1365-2567.2004.01822.x

55. Kamen LA, Schlessinger J, Lowell CA. Pyk2 Is Required for Neutrophil Degranulation and Host Defense Responses to Bacterial Infection. J Immunol (2011) 186:1656-65. doi: 10.4049/jimmunol.1002093

56. Groh V, Bahram S, Bauer S, Herman A, Beauchamp M, Spies T. Cell StressRegulated Human Major Histocompatibility Complex Class I Gene Expressed in Gastrointestinal Epithelium. Proc Natl Acad Sci USA (1996) 93:12445-50. doi: $10.1073 /$ pnas.93.22.12445

57. Chen RS, Deng TC, Garcia T, Sellers ZM, Best PM. Calcium Channel Gamma Subunits: A Functionally Diverse Protein Family. Cell Biochem Biophys (2007) 47:178-86. doi: 10.1007/s12013-007-0002-0

58. Emmrich S, Henke K, Li Z, Schoning J, Schambach A, Reinhardt D, et al. Deciphering the Role of Mir-99 Similar to 125 Clusters in the Hematopoietic System. Blood (2011) 118:99-9. doi: 10.1182/blood.V118.21.213.213

59. Emmrich S, Rasche M, Schöning J, Reimer C, Keihani S, Maroz A, et al. miR99a/100 125b Tricistrons Regulate Hematopoietic Stem And Progenitor Cell Homeostasis By Shifting The Balance Between TGF $\beta$ and Wnt Signaling. Genes Dev (2014) 28(8):858-74. doi: 10.1101/gad.233791.113

60. Khalaj M, Woolthuis CM, Hu WH, Durham BH, Chu SH, Qamar S, et al. miR-99 Regulates Normal and Malignant Hematopoietic Stem Cell SelfRenewal. J Exp Med (2017) 214:2453-70. doi: 10.1084/jem.20161595

61. Sun DD, Lee YS, Malhotra A, Kim HK, Matecic M, Evans C, et al. miR-99 Family of MicroRNAs Suppresses the Expression of Prostate-Specific Antigen and Prostate Cancer Cell Proliferation. Cancer Res (2011) 71:1313-24. doi: 10.1158/0008-5472.CAN-10-1031

62. Xin JX, Yue Z, Zhang S, Jiang ZH, Wang PY, Li YJ, et al. miR-99 Inhibits Cervical Carcinoma Cell Proliferation by Targeting TRIB2. Oncol Lett (2013) 6:1025-30. doi: 10.3892/ol.2013.1473 
63. Jing PY, Zhao N, Ye MX, Zhang Y, Zhang ZP, Sun JY, et al. Protein Arginine Methyltransferase 5 Promotes Lung Cancer Metastasis via the Epigenetic Regulation of miR-99 Family/FGFR3 Signaling. Cancer Lett (2018) 427:38-48. doi: 10.1016/j.canlet.2018.04.019

64. Mueller AC, Sun D, Dutta A. The miR-99 Family Regulates the DNA Damage Response Through Its Target SNF2H. Oncogene (2013) 32:1164-72. doi: 10.1038/onc.2012.131

65. Ramasamy S, Velmurugan G, Rekha B, Anusha S, Rajan KS, Shanmugarajan S, et al. Egr-1 Mediated Cardiac miR-99 Family Expression Diverges Physiological Hypertrophy From Pathological Hypertrophy. Exp Cell Res (2018) 365:46-56. doi: 10.1016/j.yexcr.2018.02.016

66. Guissart C, Latypova X, Rollier P, Khan TN, Stamberger H, McWalter K, et al. Dual Molecular Effects of Dominant RORA Mutations Cause Two Variants of Syndromic Intellectual Disability With Either Autism or Cerebellar Ataxia. Am J Hum Genet (2018) 102:744-59. doi: 10.1016/j.ajhg.2018.02.021

67. Sun Y, Liu CH, SanGiovanni JP, Evans LP, Tian KT, Zhang B, et al. Nuclear Receptor RORalpha Regulates Pathologic Retinal Angiogenesis by Modulating SOCS3-Dependent Inflammation. Proc Natl Acad Sci USA (2015) 112:104016. doi: 10.1073/pnas.1504387112

68. Liu Y, Chen Y, Zhang J, Liu Y, Zhang Y, Su Z. Retinoic Acid Receptor-Related Orphan Receptor Alpha Stimulates Adipose Tissue Inflammation by Modulating Endoplasmic Reticulum Stress. J Biol Chem (2017) 292:1395969. doi: 10.1074/jbc.M117.782391

69. Dzhagalov I, Giguere V, He YW. Lymphocyte Development and Function in the Absence of Retinoic Acid-Related Orphan Receptor Alpha. J Immunol (2004) 173:2952-9. doi: 10.4049/jimmunol.173.5.2952

70. Delerive P, Monte D, Dubois G, Trottein F, Fruchart-Najib J, Mariani J, et al. The Orphan Nuclear Receptor ROR Alpha Is a Negative Regulator of the Inflammatory Response. EMBO Rep (2001) 2:42-8. doi: 10.1093/embo-reports/kve007

71. Oh SK, Kim D, Kim K, Boo K, Yu YS, Kim IS, et al. ROR Alpha Is Crucial for Attenuated Inflammatory Response to Maintain Intestinal Homeostasis. Proc Natl Acad Sci USA (2019) 116:21140-9. doi: 10.1073/pnas.1907595116

72. Haimovich B, Calvano J, Haimovich AD, Calvano SE, Coyle SM, Lowry SF. In Vivo Endotoxin Synchronizes and Suppresses Clock Gene Expression in Human Peripheral Blood Leukocytes. Crit Care Med (2010) 38:751-8. doi: 10.1097/CCM.0b013e3181cd131c

73. Houseright RA, Rosowski EE, Lam PY, Tauzin SJM, Mulvaney O, Dewey CN, et al. Cell Type Specific Gene Expression Profiling Reveals a Role for
Complement Component C3 in Neutrophil Responses to Tissue Damage. Sci Rep (2020) 10:15716. doi: 10.1038/s41598-020-72750-9

74. Adrover JM, Del Fresno C, Crainiciuc G, Cuartero MI, Casanova-Acebes M, Weiss LA, et al. A Neutrophil Timer Coordinates Immune Defense and Vascular Protection. Immunity (2019) 50:390-402 e10. doi: 10.1016/ j.immuni.2019.01.002

75. Ren DL, Zhang JL, Yang LQ, Wang XB, Wang ZY, Huang DF, et al. Circadian Genes Period1b and Period2 Differentially Regulate Inflammatory Responses in Zebrafish. Fish Shellfish Immunol (2018) 77:139-46. doi: 10.1016/ j.fsi.2018.03.048

76. Chellappan DK, Yee LW, Xuan KY, Kunalan K, Rou LC, Jean LS, et al. Targeting Neutrophils Using Novel Drug Delivery Systems in Chronic Respiratory Diseases. Drug Dev Res (2020) 81(4):419-436. doi: 10.1002/ ddr. 21648

77. Mejias JC, Forrest OA, Margaroli C, Frey Rubio DA, Viera L, Li J, et al Neutrophil-Targeted, Protease-Activated Pulmonary Drug Delivery Blocks Airway and Systemic Inflammation. JCI Insight (2019) 4(23):e131468. doi: 10.1172/jci.insight.131468

78. Chu D, Dong X, Shi X, Zhang C, Wang Z. Neutrophil-Based Drug Delivery Systems. Adv Mater (2018) 30:e1706245. doi: 10.1002/adma.201706245

Conflict of Interest: The authors declare that the research was conducted in the absence of any commercial or financial relationships that could be construed as a potential conflict of interest.

Publisher's Note: All claims expressed in this article are solely those of the authors and do not necessarily represent those of their affiliated organizations, or those of the publisher, the editors and the reviewers. Any product that may be evaluated in this article, or claim that may be made by its manufacturer, is not guaranteed or endorsed by the publisher.

Copyright (C) 2022 Hsu, Wang, Syahirah, Liu, Li, Zhang, Wang, Cao, Tian, Matosevic, Staiger, Wan and Deng. This is an open-access article distributed under the terms of the Creative Commons Attribution License (CC BY). The use, distribution or reproduction in other forums is permitted, provided the original author(s) and the copyright owner(s) are credited and that the original publication in this journal is cited, in accordance with accepted academic practice. No use, distribution or reproduction is permitted which does not comply with these terms. 